\title{
Effects of visual span on Chinese reading performance in normal peripheral vision
}

Lyu A ${ }^{\mathrm{a}}$, Silva $\mathrm{AE}^{\mathrm{b}}$, Cheung $\mathrm{SH}^{\mathrm{c}}$, Thompson $\mathrm{B}^{\mathrm{b}, \mathrm{e}}$, Abel L ${ }^{\mathrm{d}}$, Cheong AMY ${ }^{\mathrm{a}, \mathrm{e}, \mathrm{f}}$

${ }^{a}$ School of Optometry, The Hong Kong Polytechnic University, Hong Kong

${ }^{\mathrm{b}}$ School of Optometry and Vision Science, University of Waterloo, ON, Canada

${ }^{c}$ Department of Psychology, The University of Hong Kong, Hong Kong

${ }^{\mathrm{d}}$ School of Medicine, Deakin University, Burwood, VIC, Australia

${ }^{\mathrm{e}}$ Centre for Eye and Vision Research Limited, Hong Kong

${ }^{\mathrm{f}}$ Research Centre for SHARP Vision, The Hong Kong Polytechnic University, Hong Kong

Correspondence's Address: Allen MY Cheong

School of Optometry, The Hong Kong Polytechnic University, Hung Hom, Hong Kong

Telephone:

852-2766-6108

Facsimile:

$852-2764-6051$

Electronic mail:

allen.my.cheong@polyu.edu.hk 


\begin{abstract}
The current study examined the relationships among temporal processing speed, spatial visual span and Chinese character reading speed in normal central and peripheral vision. Maximum reading speed (MRS) and critical print size (CPS) of 26 native Chinese readers (13 young and 13 older adults) were determined at three visual field locations: central vision, $10^{\circ}$ left and $10^{\circ}$ below fixation using a rapid serial visual presentation (RSVP) task. Temporal processing speed was measured using trigrams of randomly selected Chinese characters presented at a range of exposure durations, while spatial visual span was measured using trigrams presented at different spatial positions. It was found that shorter temporal processing speed and larger spatial visual span were associated with faster MRS at the central and inferior visual field locations, but not at the left of fixation location. As expected, reading and visual span metrics were better in central vision compared to both peripheral locations. In addition, reading, temporal processing, and spatial visual span metrics were better in the young than older subjects (except for similar temporal processing speed at two peripheral locations). The results for central and inferior presentation locations support the hypothesis that temporal processing speed and spatial visual span were associated with Chinese character reading speed. Surprisingly, no correlation was observed for the $10^{\circ}$ left of the fixation location, suggesting that the factors affecting reading speed might differ for inferior and lateral peripheral viewing locations.
\end{abstract}

Keywords: visual span; RSVP; Chinese reading; peripheral vision. 


\section{Introduction}

Reading relies on high-resolution central vision, and reading difficulty is a major concern for patients with central scotomas caused by age-related macular degeneration (AMD), who are forced to rely on para-central or peripheral vision. AMD is common with a prevalence of $4.9 \%$ in adults over 50 years old age in China (Jin, Zou, Chen, Zhang, Young, Wang \& Zheng, 2019) and 7.51\% in adults over 70 years old in Hong Kong (Wang, Ng, Lai $\&$ Wong, 2019). Studies of peripheral reading involving individuals with normal vision have provided insights into the factors that limit reading in patients with AMD (refer to (Chung, 2010) for a review). For example, reading in the peripheral visual field is impaired due to reduced visual acuity (Chung, Mansfield \& Legge, 1998) and crowding (Coates, Chin \& Chung, 2013, Jacobs, 1979) (i.e. difficulty in differentiating visual objects in close proximity) relative to central vision. Chung and colleagues examined reading speed across different eccentricities in six young adults with normal vision and reported that the maximum reading speed decreased from an average of $807 \mathrm{wpm}$ with foveal reading to around $237 \mathrm{wpm}$ at $10^{\circ}$ below fixation (Chung et al., 1998). The slow reading speed for peripheral vision has been attributed to various causes, including slower lexical processing (Lee, Legge \& Ortiz, 2003), poorer eye movement control (Bethlehem, Dumoulin, Dalmaijer, Smit, Berendschot, Nijboer \& Van der Stigchel 2014; Bullimore \& Bailey, 1995; Whittaker, Cummings \& Swieson, 1991), and a restricted peripheral visual span (Legge, Mansfield \& Chung, 2001).

Visual span has been shown to strongly correlate with English reading speed (Chung et al., 1998; Kwon, Legge \& Dubbels, 2007; Legge, Cheung, Yu, Chung, Lee \& Owens, 2007; Legge, Hooven, Klitz, Stephen Mansfield \& Tjan, 2002). It is defined as the number of letters that can be recognized accurately on either side of a single fixation at a glance (Legge, Ahn, Klitz \& Luebker, 1997). Spatial visual span (SVS) is examined by presenting three unrelated English letters (a trigram) at different positions or away from the fixation at a fixed exposure duration (Fig. 1a). Proportion of recognition accuracy is plotted against each letter position, combining across trials with each position occupied by the left, middle and right letter of a trigram. The width of the spatial position yielding $80 \%$ accuracy is computed (Fig. $1 b)$. 
Fig. 1 Measurement of spatial visual span (SVS) using a trigram of 3 English letters

(a)

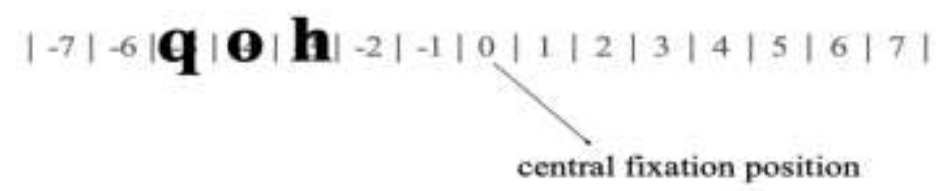

(b)

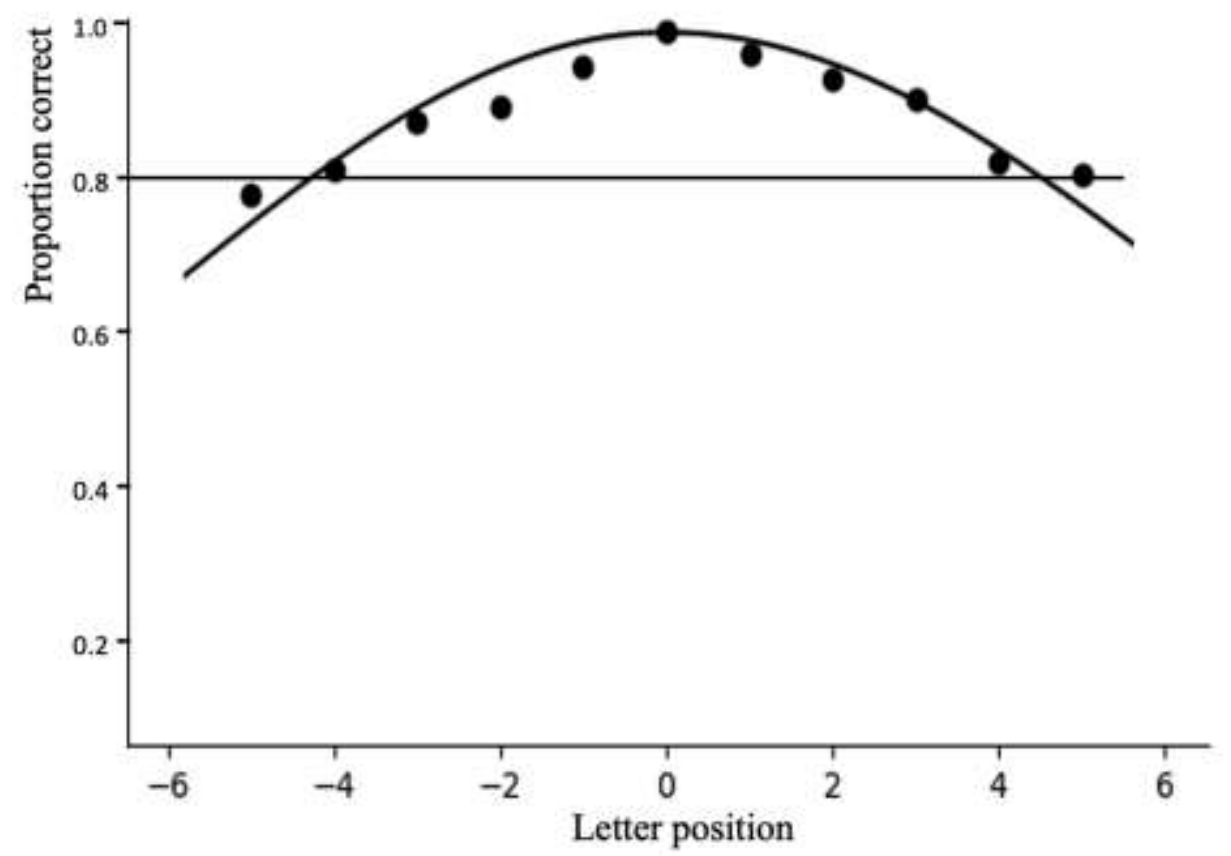

(a) Three unrelated English letters are presented at different letter positions for a fixed exposure duration. Participants are asked to recognize all three letters from left to right order while fixating at the central position.

(b) Proportion of recognition accuracy is plotted against each letter position, combining across trials, regardless of whether it is the left, middle or right letter of a trigram. The horizontal line represents the $80 \%$ threshold of the recognition accuracy. 
SVS was estimated to be 10 letters (five letter positions each to the left and the right of fixation) in normally sighted English readers, but dropped to 1.7 letters when reading at $15^{\circ}$ below fixation (Legge et al., 2001). The reduced visual span size in normal peripheral vision was consistent with the reduction of reading speed reported by Chung et al. who found that reading speed decreased from $807 \mathrm{wpm}$ in the fovea to around $174 \mathrm{wpm} 15^{\circ}$ below fixation (Chung et al., 1998). To further examine the association between reading speed and SVS, subsequent studies examined whether training with peripheral trigram recognition could improve peripheral reading performance. For example, Chung and colleagues recruited 18 young native English readers and trained them on a trigram-recognition task at $10^{\circ}$ below fixation for four days. SVS increased after training and this improvement was correlated with faster peripheral reading speed (Chung, Legge \& Cheung, 2004). Similar training effects were also observed in older adults with normal peripheral vision (Yu, Cheung, Legge \& Chung, 2010). These findings suggest a link between SVS and English reading speed for all ages for both central and peripheral vision (Chung et al., 1998; Kwon et al., 2007; Legge et al., 2007; Legge et al., 2002).

In addition to the spatial visual span, temporal processing speed is also associated with English reading performance (Cheong, Legge, Lawrence, Cheung \& Ruff, 2007; Cheong, Legge, Lawrence, Cheung \& Ruff, 2008; Chung et al., 2004; Chung, 2021; Yu et al., 2010). Temporal processing speed is quantified by presenting trigrams at different exposure durations. Threshold is determined as the exposure duration that yields $80 \%$ accuracy for middle letter recognition, known as the temporal trigram threshold (TTT). Cheong et al. found that the mean threshold was $97.0 \pm 34.2 \mathrm{~ms}$ and less than $13 \mathrm{~ms}^{\mathrm{a}}$ at $10^{\circ}$ below fixation and central vision respectively, indicating that the temporal processing speed for letter identification was slower in peripheral vision compared to central vision (Cheong et al., 2007). Furthermore, Yu et al. investigated whether enhancing temporal processing speed could improve the reading speed at peripheral vision. They found that four days of intensive training on the TTT task resulted in a significant improvement in RSVP reading speed and shorter TTT in peripheral vision (Yu, Legge, Wagoner \& Chung, 2018).

\footnotetext{
a Temporal trigram threshold could not be precisely determined for normal vision subjects at central vision because of the limitation of the refresh rate of monitor $(76 \mathrm{~Hz})$.
} 
Despite extensive research examining the association between visual span and reading of English, few studies have investigated the correlation between visual span and Chinese character reading performance. Unlike phonics-based languages that are written using a small number of letters with simple spatial forms, Chinese contains an enormous number of characters (approximately 2500 frequently used Chinese characters as reported in Zhu, $\mathrm{Hu}$, Liao, Keel, Huang, Liu \& He (2019b)) with a wide range of spatial complexities arranged within a square configuration. This unique feature allows Chinese sentences to be oriented in both horizontal and vertical directions without disturbing the shape and meaning of the characters, although the former dimension is more commonly used. Different visual faculties are required for accurate recognition and fast reading of Chinese compared to English (Yeung, Ho, Chik, Lo, Luan, Chan \& Chung, 2011). Wang et al. compared SVS at central vision using stimuli of three unrelated English letters or three unrelated Chinese characters in 12 bilingual Chinese adults. Chinese trigrams were divided into three groups based on three levels of perimetric complexity. They found that the SVS decreased from 10.5 for English letters to 4.5 for high-complexity Chinese characters (Wang, He \& Legge, 2014). They concluded that crowding, which disproportionally affected reading of complex Chinese characters, was the primary factor limiting the Chinese visual span (Wang et al., 2014). In addition, two studies demonstrated a significant correlation between SVS and Chinese sentence-reading speed at central vision (Xie, Li, Zhao, Wang, Paterson, White \& Warrington, 2019; Zhu et al., 2019b). Xie and colleagues (2019) observed that SVS was agedependent, with larger (better) SVS in young subjects (5.5 for high complexity characters) compared to older subjects (3.1 characters). Zhu et al. (2019b) reported that cognitive/linguistic factors in addition to SVS contributed to Chinese sentence-reading speed, with smaller SVS size and slower reading speed for low-frequency words. A subsequent study by Zhu et al. applied a similar trigram training paradigm in English (Chung et al., 2004) to train character recognition performance at central vision in 13 native Mandarin speakers. They found that training significantly increased SVS size and Chinese sentence reading speed (Zhu, Hu, Liao, Huang, Keel, Liu \& He, 2019a).

To our knowledge, no published studies have investigated the correlation between visual span and Chinese reading performance within normal peripheral vision. This is an important knowledge gap for at least two reasons. First, unlike English letters, Chinese character trigrams are subject to two levels of crowding within peripheral vision: internal (within character) and external (between character) (Zhang, Zhang, Xue, Liu \& Yu, 2009). It 
is unclear what is the limit of visual span on Chinese reading at periphery. Second, Feng et al. reported that the crowding effect was stronger when trigrams were oriented horizontally to fixation compared to those presented vertically (Feng, Jiang \& He, 2007). However, more studies are required to confirm the benefit of vertically-oriented text on Chinese reading in peripheral vision.

In the present study, reading performance, temporal processing speed, and spatial visual span were measured at fixation and two peripheral visual field locations: left lateral and inferior visual fields, which are the two most frequent para-central locations adopted by AMD patients fixating at a central cross $(33.7 \%$ and $39 \%$ respectively) (Fletcher \& Schuchard, 1997). Two age groups were tested: young adults and older adults. We hypothesized that 1) there would be a significant association among temporal processing speed, spatial visual span and Chinese reading performance for central and peripheral visual fields; 2) central vision would show superior performance than peripheral vision. At peripheral vision, vertical presentations of Chinese characters would elicit better visual performance than horizontal presentations; 3) young subjects would have better performance than older subjects. Understanding the factors affecting Chinese reading ability in both central and peripheral visual fields could provide a platform for future development of better visual rehabilitation for low vision patients with central vision loss.

\section{Materials and methods}

\section{$\underline{2.1 \quad \text { Subjects }}$}

13 young (aged between 18 to 32 years) and 13 older (over 60 years old) healthy subjects with normal vision were recruited from The Hong Kong Polytechnic University and social media. All subjects spoke Cantonese as their first language and read traditional Chinese characters. They all had normal or corrected-to-normal vision, with distance visual acuity of 0.0 and $0.1 \log$ MAR or better for the young and older groups, respectively. Exclusion criteria included any history of ophthalmic, neurological or psychiatric illness, or medication that might affect eye movements or reading performance. Demographic information, including age, gender, educational level, and reading habits (percentage of time spent reading horizontally vs. vertically orientated Chinese text) was collected and summarized in Table 1. Informed consent was obtained in accordance with a protocol approved by the Department Research Committee of the School of Optometry of The Hong 
Kong Polytechnic University (HSEARS20190716005). The study followed the tenets of the Declaration of Helsinki.

Table 1. Demographic data of the subjects

\begin{tabular}{|c|c|c|c|}
\hline & Young $(n=13)$ & Older $(n=13)$ & p-value \\
\hline Age (years) & $\begin{array}{c}26.26 \pm 3.54 \\
(22-32)\end{array}$ & $\begin{array}{c}65.52 \pm 3.94 \\
(60-73)\end{array}$ & --- \\
\hline Gender & $\begin{array}{l}\text { F: } 38.46 \% \\
\text { M: } 61.54 \%\end{array}$ & $\begin{array}{l}\text { F: } 69.23 \% \\
\text { M: } 30.77 \%\end{array}$ & 0.24 \\
\hline Educational level & & & \\
\hline Primary school (\%) & --- & --- & --- \\
\hline Secondary school (\%) & --- & $61.5 \%$ & --- \\
\hline University or above (\%) & $100 \%$ & $38.5 \%$ & --- \\
\hline Visual acuity (logMAR) & $\begin{array}{c}\text { Distance: }-0.07 \pm 0.07 \\
\text { Near: }-0.07 \pm 0.09\end{array}$ & $\begin{array}{c}\text { Distance: } 0.04 \pm 0.06 \\
\text { Near: } 0.13 \pm 0.07\end{array}$ & $\begin{array}{l}0.0001 \\
<0.0001\end{array}$ \\
\hline $\begin{array}{l}\text { Percentage of time } \\
\text { reading Chinese text } \\
\text { orientated in horizontal } \\
\text { and vertical dimensions }\end{array}$ & $\begin{array}{c}\text { Horizontal: } 82.15 \% \\
\text { Vertical: } 17.85 \%\end{array}$ & $\begin{array}{l}\text { Horizontal: } 71.54 \% \\
\text { Vertical: } 28.46 \%\end{array}$ & 0.14 \\
\hline
\end{tabular}

LogMAR = logarithm of the minimum angle of resolution measured using the Early Treatment Diabetic Retinopathy Study (ETDRS) chart at $4 \mathrm{~m}$ (distance) and $40 \mathrm{~cm}$ (near). Mean \pm 1 standard deviation (SD) is calculated across groups.

\section{$2.2 \quad$ Experimental design}

Subjects visited The Hong Kong Polytechnic University Research Optometry Clinic on four occasions. All subjects wore full refractive correction with appropriate near additions (if needed) for different testing distances. All tests were performed on the right eye with the left eye occluded. 


\subsubsection{Reading performance}

Reading performance was measured using rapid serial visual presentation (RSVP), which involved character-by-character presentation of sentences on a screen (Chung, 2002; Chung, 2011; Chung, 2021; Legge et al., 2001). Stimuli were generated using PsychoPy 2020.1.3 (Peirce \& MacAskill, 2018) and presented on a 24-inch LCD monitor (BENQ x12540: $120 \mathrm{~Hz}$ refresh rate, 1920 x 1080 pixel resolution). The viewing distance was $120 \mathrm{~cm}$ for the central testing condition to enable the presentation of sufficiently small characters and $65 \mathrm{~cm}$ for the peripheral testing conditions. A chinrest and a forehead bar were used to stabilize head position.

One sentence for each RSVP trial was randomly selected from a pool of 605 sentences comprising 15 characters selected from third grade primary school textbooks. All sentences were validated to ensure their readability and validity. Briefly, sentence grammar and structure were evaluated by a primary school Chinese teacher and a clinical educator with a linguistic background. These 605 sentences were selected from a pool of 1500 sentences with minimal reading errors and similar reading speed examined by another group of subjects across all ages (also see Appendix I). Subjects were asked to fixate at a central fixation cross, while a short mask of "XXX" (displayed in the same size as the testing characters) was displayed against a white background to indicate the position of the stimulus for the upcoming trial (central, $10^{\circ}$ left of fixation or $10^{\circ}$ below fixation). Each character of the sentence was then presented sequentially (Fig. 2). The fixation cross remained for the peripheral testing conditions. Eye movements were monitored using an infrared video eye tracking system (Eyelink Portable Duo, SR Research, Scarborough, ONT, Canada) at 500 Hz. Trials were repeated if fixation deviated by more than $1^{\circ}$. 
Fig. 2 Rapid Serial Visual Presentation (RSVP) of sentences at three stimulus locations

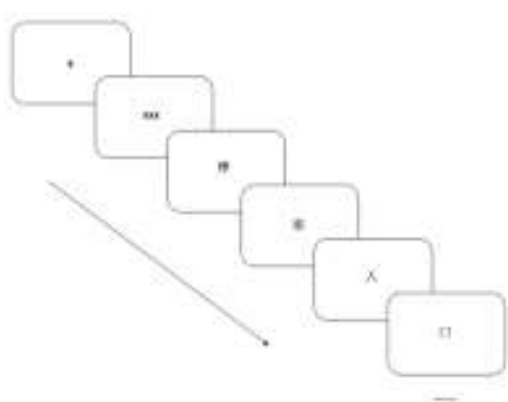

a) Central fixation

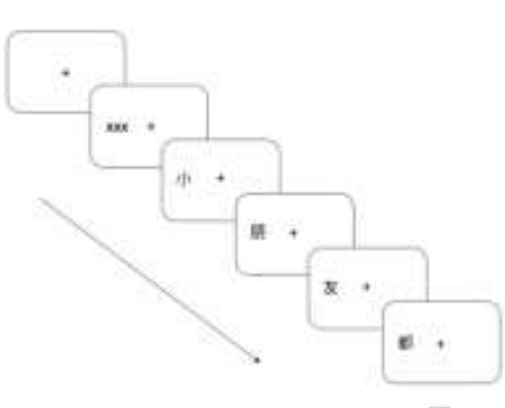

b) $10^{\circ}$ left of fixation

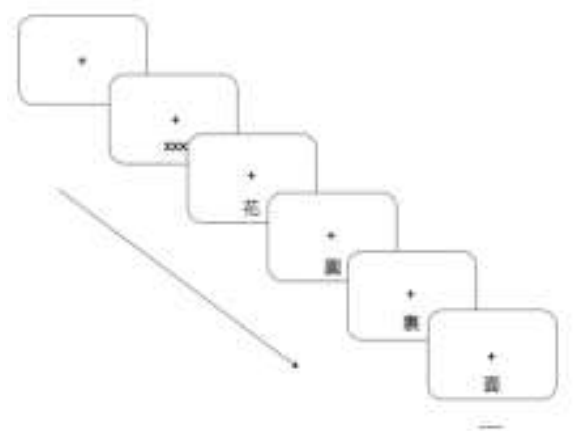

c) $10^{\circ}$ below fixation

A character-by-character sentence was presented at three different stimulus locations: (a) central vision; (b) $10^{\circ}$ left of fixation; and (c) $10^{\circ}$ below fixation. Participants were asked to read the sentence aloud. The number of the characters correctly recognized was recorded.

The full set of RSVP measurements involved at least five print sizes (defined by the vertical height of a square character configuration, ranging from $0.13^{\circ}-0.80^{\circ}$ at the fovea, and $1.26^{\circ}-5.02^{\circ}$ at $10^{\circ}$ eccentricity) presented at five or more exposure durations. Exposure duration was changed in a ratio of 1.5 times if the longest presentation speed had accuracy lower than $80 \%$ or the shortest exposure duration yielded an accuracy higher than $30 \%$. Each size and duration combination was repeated five times for a total of at least 125 individual sentences. A psychometric function (cumulative Gaussian distribution) relating proportion correct character recognition to exposure duration was fitted separately for each character size (Wichmann \& Hill, 2001a; Wichmann \& Hill, 2001b). The exposure durations that yielded $80 \%$ recognition accuracy for each print size were derived and then plotted against $\log$ reading speed (log characters per minute; $\log \mathrm{cpm}$ ) as a function of log print size (minimum angle of resolution, $\log$ MAR). A bilinear function was applied, in which reading speed was assumed to increase linearly with increasing print size before reaching a plateau. The maximum reading speed (MRS) and the smallest print size eliciting the MRS, known as the critical print size (CPS), were calculated from the bilinear fit (Fig. 3) as the primary outcome measures (Cheong et al., 2007; Chung, 2002; Chung, 2011; Chung et al., 1998; Chung, 2021). 
Fig. 3 RSVP curve fitting between print size and reading speed
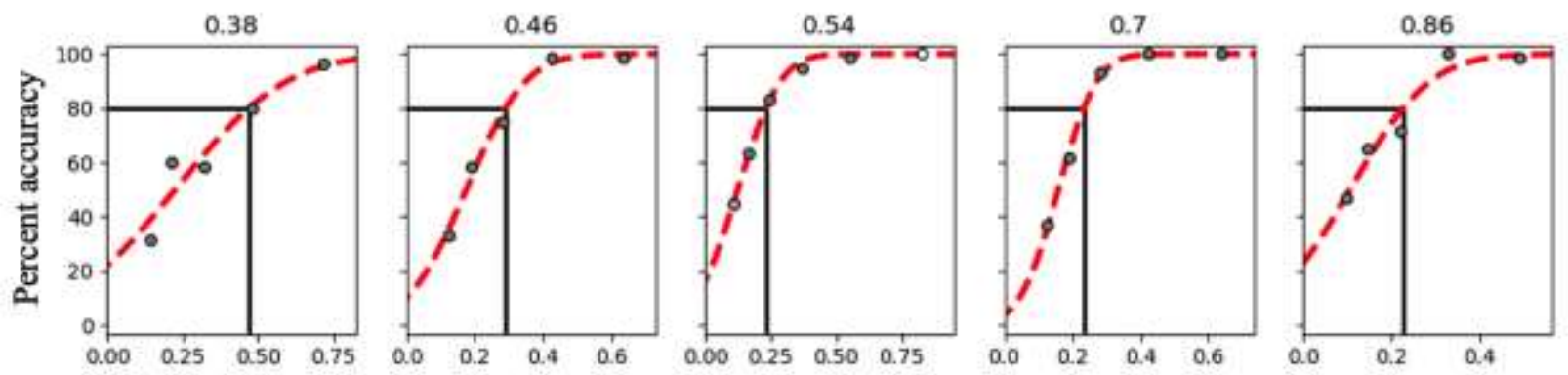

Exposure durations (s)

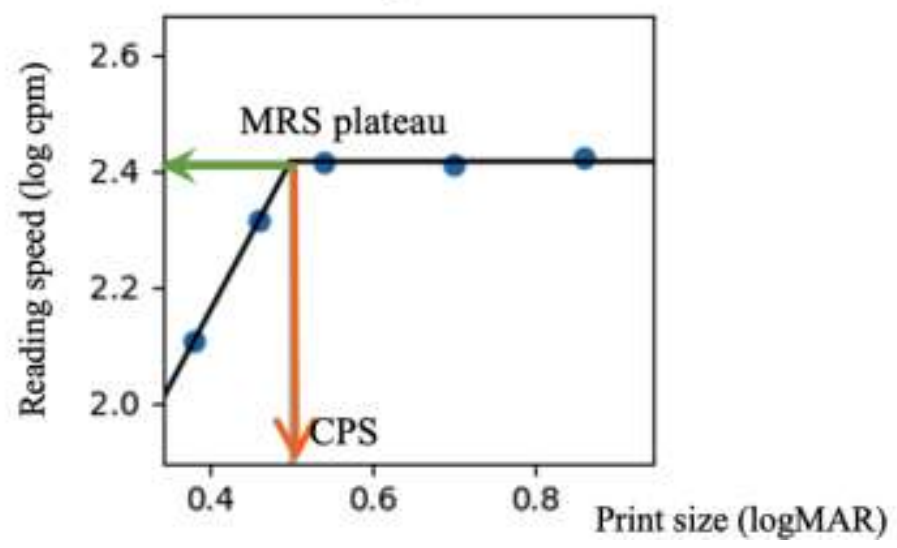

The upper panel illustrates the percent correct vs. exposure duration for five print sizes $(0.38$, 0.46, 0.54, 0.7 and 0.86 logMAR) for RSVP measurement at central vision of a normallysighted old subject. The dots represent the percent correct for a specific exposure duration, while the red curve represents the psychometric function (cumulative Gaussian distribution). The vertical dark line represents the exposure time that yielded $80 \%$ correct character recognition.

The lower panel illustrates the log reading speed (log characters per minute) (i.e. blue dots) versus a range of print sizes, in which the dots are fitted with a bilinear function. The maximum reading speed (MRS) (indicated as the green horizontal arrow) and critical print size (CPS) (indicated as the orange vertical arrow) were determined.

\subsubsection{Temporal processing speed}

The TTT (temporal trigram threshold) test was used to examine the temporal processing speed. Stimuli were generated using GNU Octave Version 4.2.2 (Octave, 2018) in Lubuntu 18.04 and projected on a 24-inch LCD monitor (BENQ x12411). The refresh rate was $60 \mathrm{~Hz}$. The font size was personalized for each participant, being 1.5 times larger than 
RSVP-calculated CPS at the corresponding visual location (Chung, 2002). Central TTT was tested first followed by the two peripheral locations in randomized order. Both horizontal and vertical trigram recognition accuracy were assessed at central vision. Subjects were asked to fixate the mid-point between two vertically/horizontally separated dark fixation dots, while three unrelated Chinese characters appeared horizontally/vertically for a range of exposure durations. They were asked to recognize all three characters in an order from left to right (horizontal trigram) (see Fig. 4a) or from top to bottom (vertical trigram) (see Fig. 4b). After the fixation dots disappeared, participants were asked to select the individual recognized character from a pool of 20 characters by clicking a mouse. In the two peripheral visual locations, subjects fixated at a dot on the screen, while identifying all three characters displayed either horizontally at $10^{\circ}$ below or vertically at $10^{\circ}$ left of the fixation dot (Fig. 4c and d). They were asked to select the individual recognized character after the fixation dot disappeared. Eye movements were visually monitored using an external web-camera (Logitech QuickCam Pro 5000), and any trial with visible eye movements (i.e. pupil moved in the camera image during the trial) was replaced by a new trial. Previous research showed that this method could reliably detect saccades of $2^{\circ}$ (Cheong et al., 2007). Due to different programming systems of the tests, the same eye tracker used for RSVP was not employed for the TTT test. For each session, pre-defined five exposure durations were tested, with the shortest and longest exposure durations being 0.5 times and 2 times of 60 (seconds) divided by individual RSVP MRS (cpm) at the corresponding visual locations. Additional exposure duration was added in a ratio of 1.5 times if the initial duration was too long or too short to elicit between $20-80 \%$ identification accuracy. Thirty-six trials were presented during each exposure period. The relationships between identification accuracy and exposure speeds were fitted to a psychometric function (cumulative Gaussian distribution). The temporal threshold was defined as the exposure duration that produced $80 \%$ recognition accuracy of the middle character from each trigram (Cheong et al., 2007). Due to the limitation of the display monitor's refresh rate, the minimum exposure duration of presented stimuli was $16.7 \mathrm{~ms}$. While this could potentially limit measuring the temporal threshold, for subjects whose recognition accuracy was equal to or reached more than $80 \%$ at $16.7 \mathrm{~ms}$, this exposure duration was used as their temporal threshold. The threshold exposure duration was exported as an outcome measure for group analysis. 
Fig. 4 Temporal trigram threshold (TTT) tests at three stimulus locations using three unrelated Chinese characters

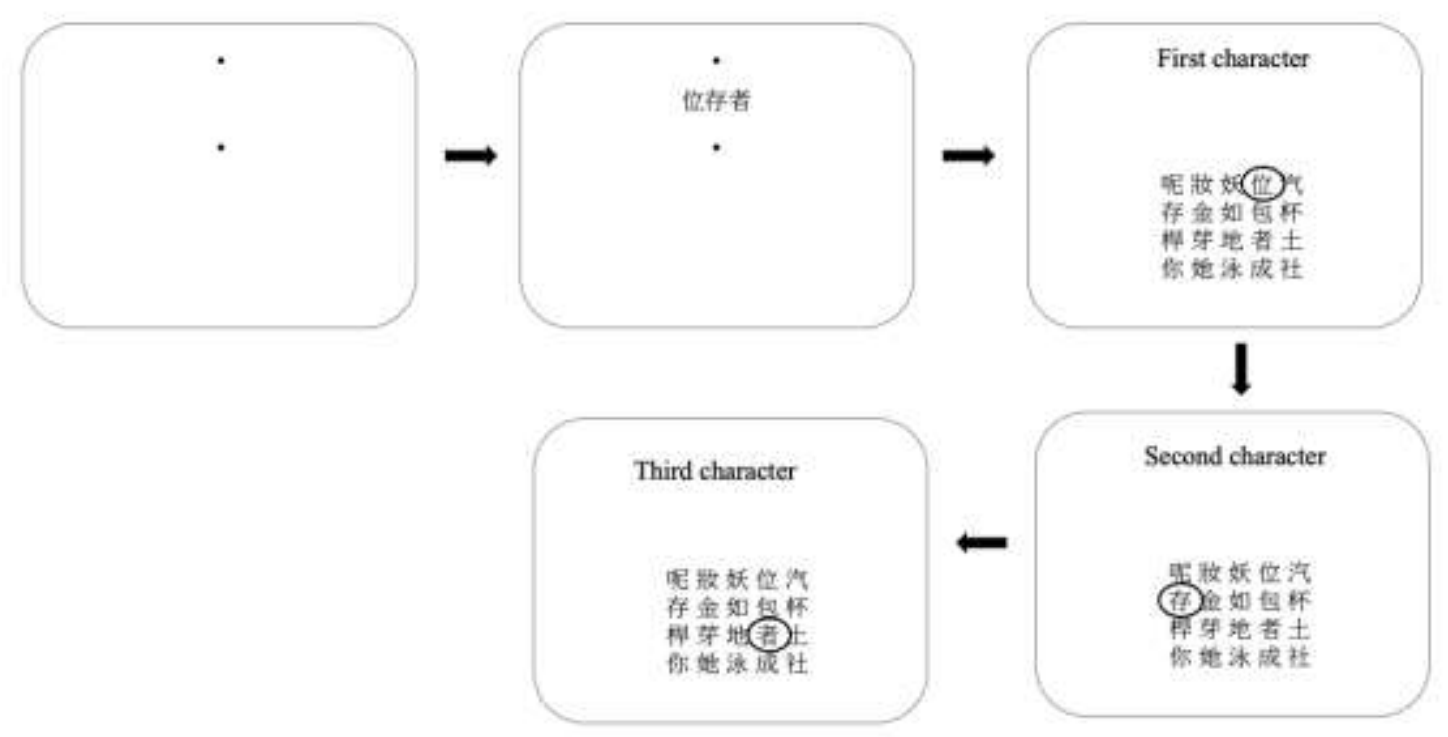

a) central TTT test (horizontal presentation)

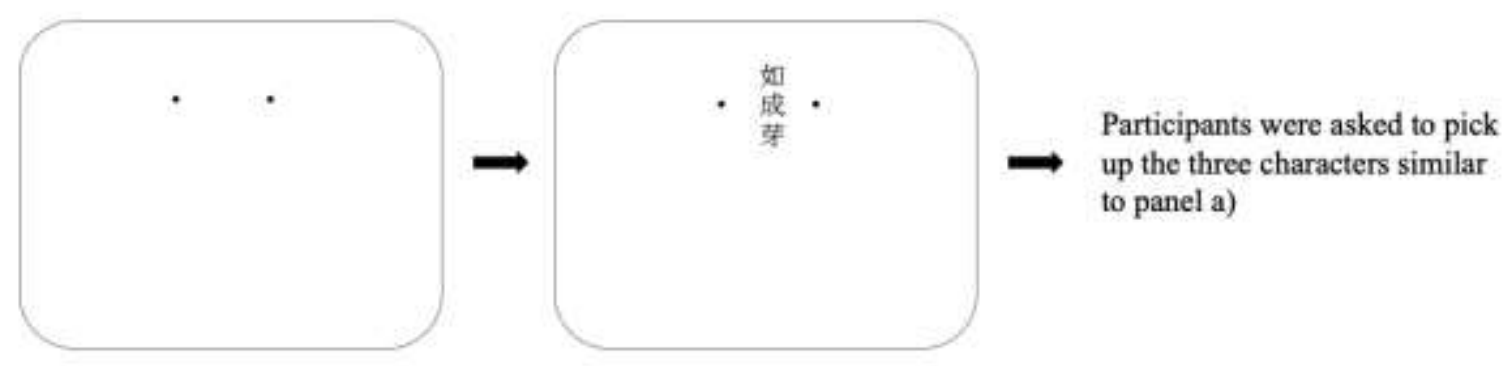

b) central TTT test (vertical presentation)

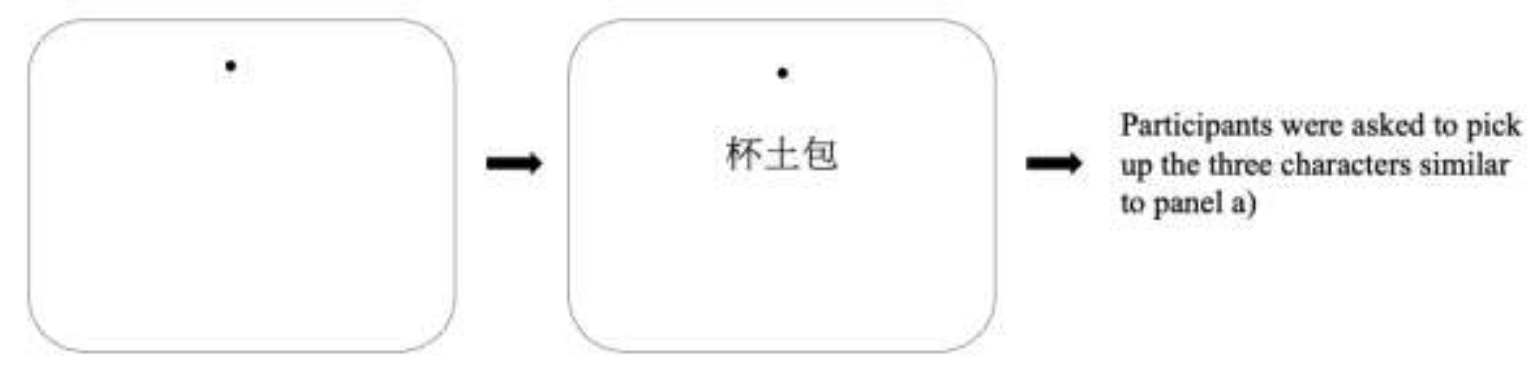

c) inferior TTT test (horizontal presentation)

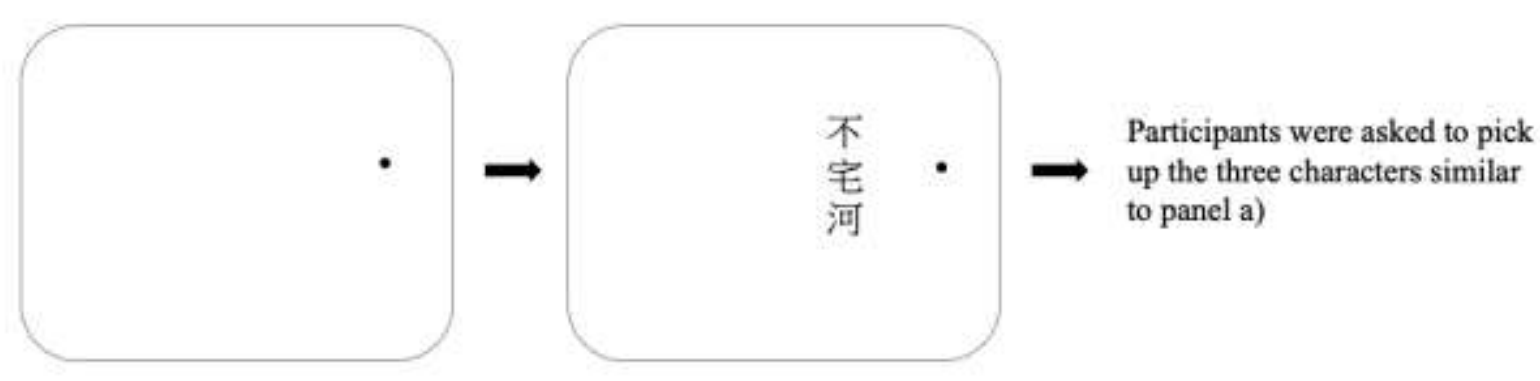

d) left TTT test (vertical presentation) 
Three unrelated Chinese characters are presented for four visual conditions: (a) central vision (horizontal presentation); (b) central vision (vertical presentation); (c) $10^{\circ}$ lower vision; and (d) $10^{\circ}$ left vision for different exposure durations. For the central TTT test, participants were asked to fixate at the middle position of the two dots and recognize the characters. For $10^{\circ}$ inferior and left TTT tests, participants were asked to fixate on a single black dot and recognize the characters.

\subsubsection{Spatial visual span}

Methods for the spatial visual span test (SVS) were identical to the TTT task, with a print size of 1.5 times larger than the individual RSVP CPS at the corresponding visual fields. However, instead of presenting three characters at different exposure durations, trigrams were presented for a fixed duration $(0.2 \mathrm{~s})$ at five different spatial positions to the left and right (or top and bottom) of the central position, resulting in a total of 11 displayed positions (Fig. 5). Sixteen trials were repeated for each position. Proportion correct of recognition accuracy was computed at each displayed position, combining across the trials regardless of whether it was the first, middle or last character within a trigram. An individual SVS profile was plotted as a function of spatial positions and fitted with split Gaussians. The area under the individual Gaussian curves (AUC) was calculated to quantify the entire SVS profile. The displayed positions that achieved $80 \%$ accuracy or more of the fitted curve were estimated for each subject. Furthermore, individuals' SVS performance was expressed as the average percent correct of character recognition at the central five displayed positions and AUC for group analysis. 
Fig. 5 Spatial visual span (SVS) tests at three stimulus locations using three unrelated Chinese characters

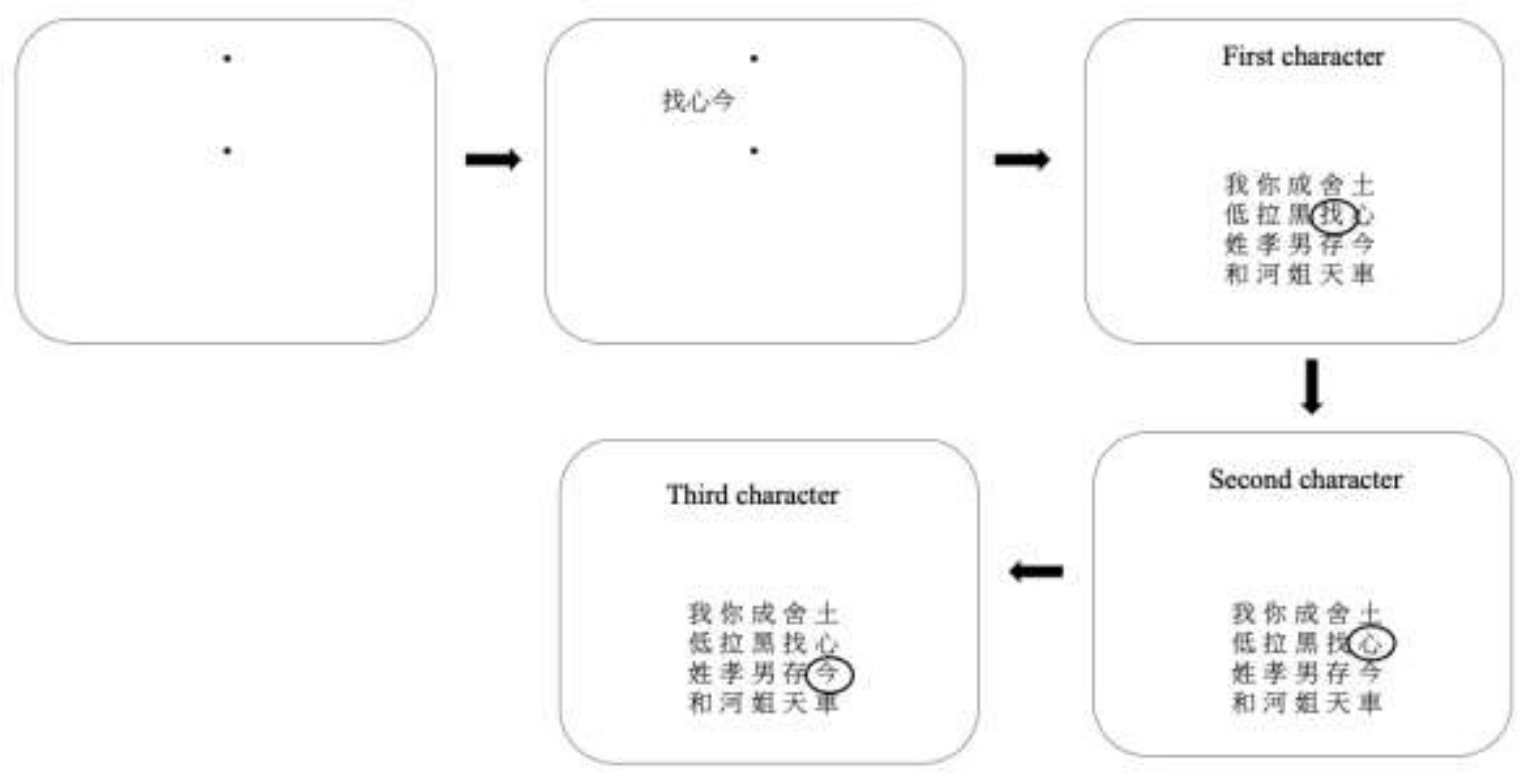

a) central SVS test (horizontal presentation)

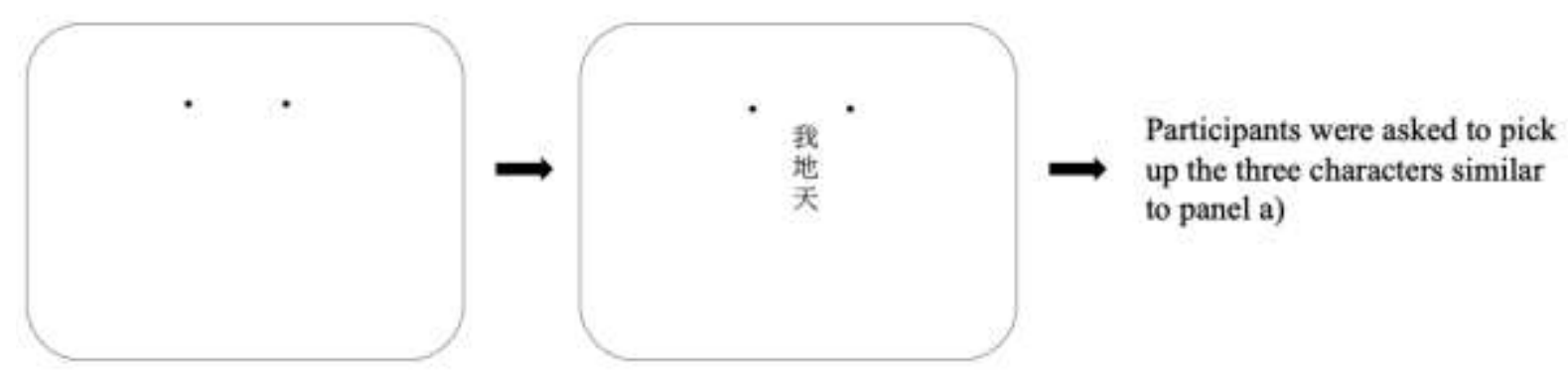

b) central SVS test (vertical presentation)

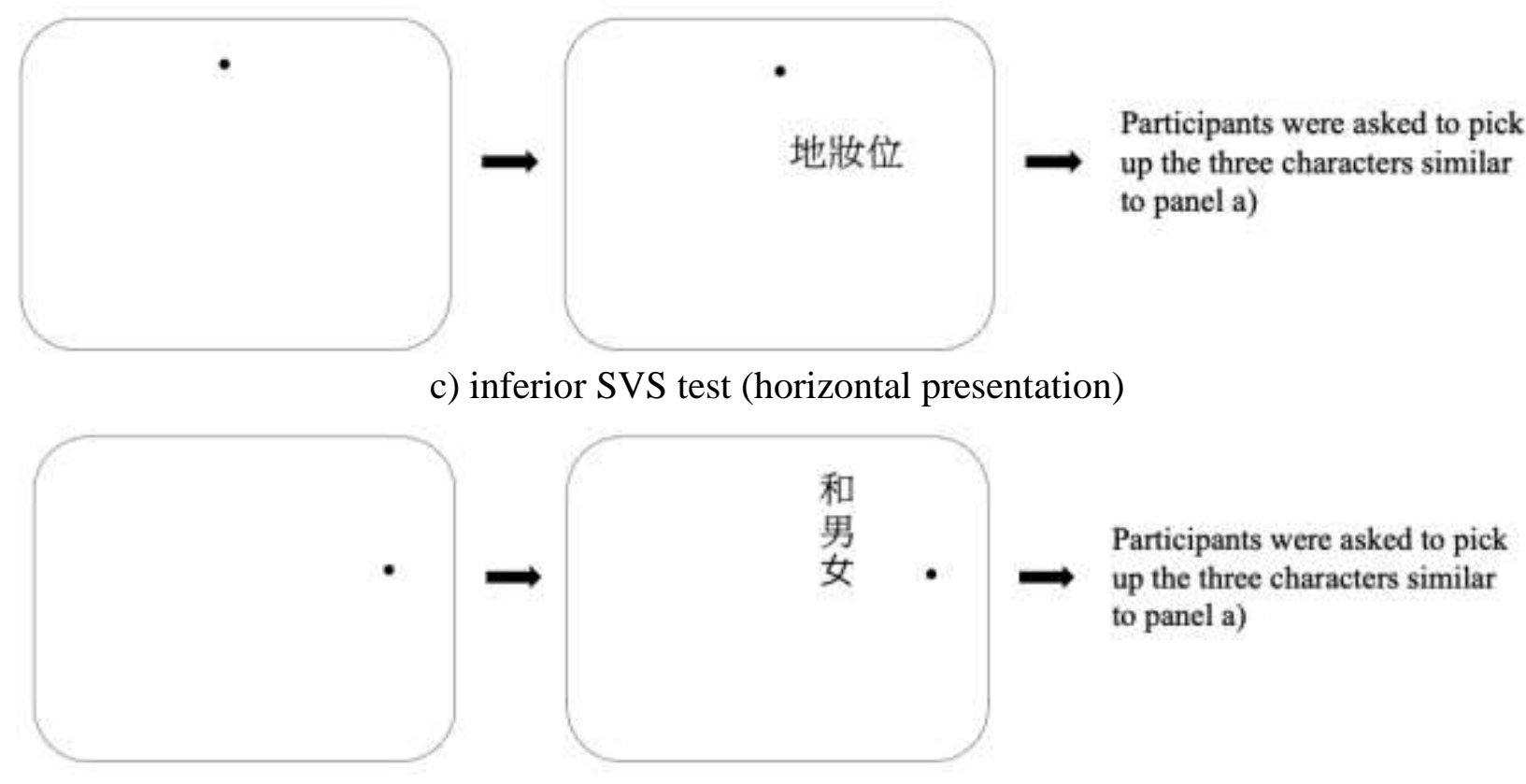

d) left SVS test (vertical presentation) 
SVS was tested for four visual conditions: (a) central vision (horizontal presentation); (b) central vision (vertical presentation); (c) $10^{\circ}$ lower vision; and (d) $10^{\circ}$ left vision. For the central SVS test, participants were asked to fixate at the middle position of the two dots and recognize the characters. For $10^{\circ}$ left and lower SVS tests, participants were asked to fixate on the black dot and recognize the characters. The trigram was presented at different displayed positions for a fixed exposure duration (0.2 s).

\section{$\underline{2.3 \quad \text { Data analysis }}$}

All group analyses were performed using GraphPad Prism 9.2.0 for Windows 64-bit, GraphPad software, San Diego, California USA, www.graphpad.com. Results of TTT were $\log$ transformed so that its frequency distribution was not significantly different from normal (Kolmogorov-Smirnov goodness of fit test, $\mathrm{p}>0.05$ ). The RSVP CPS and MRS were analysed using analysis of variance (ANOVA) with stimulus location (central vs. inferior vs. left) as within-subject factor, and age (young vs. old) as between-subject factor. Log TTT, AUC and spatial recognition accuracy outcome measures were analysed with eccentricity (central vs. $10^{\circ}$ peripheral) and text orientation (horizontal vs. vertical) as within-subject factors and age as between-subject factor. Furthermore, simple linear regressions were used to examine the contribution of temporal processing speed (log TTT) and spatial visual span (AUC and recognition accuracy in terms of the central 5-character positions) on the reading speed (i.e. RSVP MRS) at the three visual locations. Any significance was examined using Bonferroni multiple comparison post hoc tests. Adjusted p-value of less than 0.05 was considered statistically significant.

\section{Results}

\subsection{RSVP reading performance}

Chinese reading performance at different conditions was compared in terms of CPS and MRS. RSVP CPS was significantly different among the three stimulus locations (F(2, $48)=1242, \mathrm{p}<0.001)$ and between ages $(\mathrm{F}(1,24)=29.84, \mathrm{p}<0.001)$. No significant interaction effect was found $(\mathrm{F}(2,48)=2.12, \mathrm{p}=0.13)$. As expected, larger print sizes were required to achieve MRS in peripheral vision than central vision. CPS at the two peripheral locations was approximately 2.6 times larger than that at central vision $(0.58 \pm 0.11,1.51 \pm 0.08$ and $1.48 \pm$ $0.07 \log$ MAR (mean $\pm 1 \mathrm{SD}$ ) for central, inferior and left visual locations respectively, $\mathrm{p}<0.001)$. No significant CPS difference was found between left and inferior RSVP ( $=0.71)$. 
Young subjects had a smaller CPS compared to older subjects $(0.51 \pm 0.11$ vs. $0.65 \pm 0.07$ $\log$ MAR and $1.46 \pm 0.06$ vs. $1.53 \pm 0.07$ for young vs. older group at central and peripheral locations, respectively).

Similar results were found for MRS, with significant effects of stimulus location $(\mathrm{F}(2$, $48)=79.49, \mathrm{p}<0.001)$ and age $(F(1,24)=19.83$, p<0.001, Fig. 6), while the interaction between the stimulus location and age was marginally significant $(F(2,48)=2.93, p=0.06)$. MRS at central vision was around 2.5 times quicker than that at $10^{\circ}$ peripheral vision $(510.03 \pm$ 229.38, $202.91 \pm 126.08$ and $202.45 \pm 109.61$ characters per minute (cpm) (mean $\pm 1 \mathrm{SD}$ ) for central, inferior and left visual fields, respectively, $\mathrm{p}<0.001)$. Similar to CPS, left and inferior RSVP exhibited no significant difference in MRS ( $>>0.99)$. Young subjects had quicker MRS than older subjects by $261.71 \pm 283.43,155.82 \pm 126.64$ and $134.35 \pm 113.08 \mathrm{cpm}$ at central, inferior and left visual locations respectively.

Fig. 6 RSVP maximum reading speed (MRS) at three stimulus locations for young and older groups

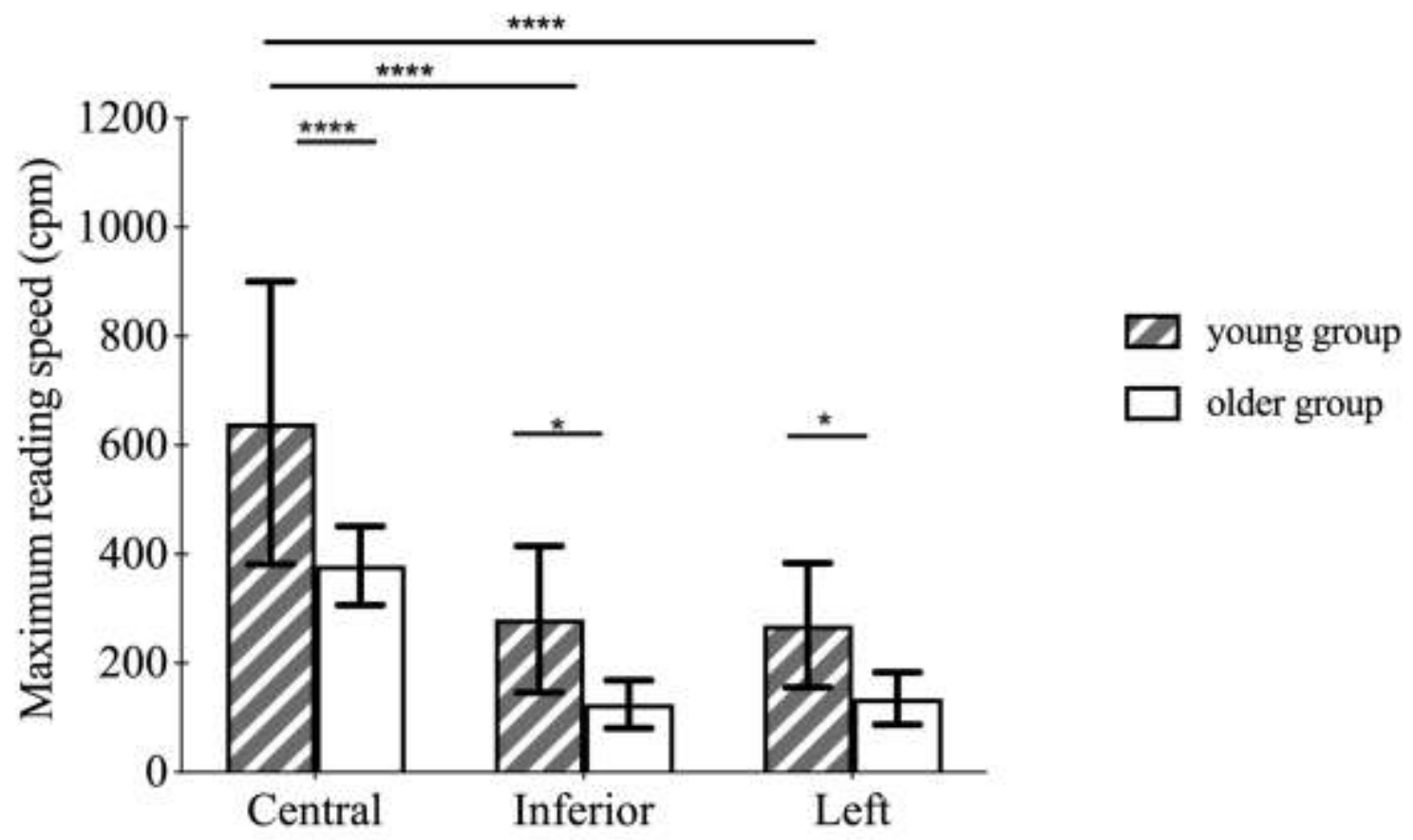

Stimulus location 
The RSVP MRSs for young and older groups at central, $10^{\circ}$ below and left fixation. Each bar represents the average MRS with \pm 1 standard deviation as the error bar. Black horizontal lines are the significant differences among the three stimuli locations or the significant differences between groups: *: $p<0.05 ; * * * *: p<0.001$.

\subsection{Temporal trigram thresholds (TTT)}

In general, recognition accuracy increased with exposure duration. However, half of the young subjects and two older subjects achieved $80 \%$ or above accuracy for recognizing horizontally presented stimuli in central vision at the shortest exposure time of $16.7 \mathrm{~ms}$ (one video frame at the $60 \mathrm{~Hz}$ frame rate). For these subjects, $16.7 \mathrm{~ms}$ represents the lower-bound of the TTT. Significant main effects of eccentricity $(F(1,24)=227,50, p<0.001)$, text orientation $(\mathrm{F}(1,24)=7.74, \mathrm{p}=0.01)$ and age $(\mathrm{F}(1,24)=10.47, \mathrm{p}=0.004)$ were found on TTT (Fig. 7). In addition, a significant interaction effect between eccentricity and age was found $(\mathrm{F}(1,24)=5.39, \mathrm{p}=0.03)$. No interaction effect between age and text orientation, eccentricity and text orientation, or among eccentricity, text orientation, and age was found $(F(1,24)<1.02, p>0.32)$. Shorter exposure durations were needed to identify the trigrams presented centrally than peripherally $(1.81 \pm 0.53$ vs. $3.14 \pm 0.27 \log \mathrm{ms})$. In addition, text presented horizontally had shorter TTT than text presented vertically $(2.42 \pm 0.80$ vs. $2.53 \pm$ $0.78 \log \mathrm{ms}$ ). Young subjects needed a shorter exposure duration to achieve $80 \%$ recognition accuracy than older subjects at central vision $(1.54 \pm 0.47$ vs. $2.08 \pm 0.44 \log \mathrm{ms}, \mathrm{p}<0.02)$, whereas no age difference was found at the peripheral locations $(3.07 \pm 0.32$ vs. $3.20 \pm 0.20$ $\log \mathrm{ms}, \mathrm{p}>0.99)$. 
Fig. 7 Temporal trigram threshold (TTT) at different visual conditions for young and older groups

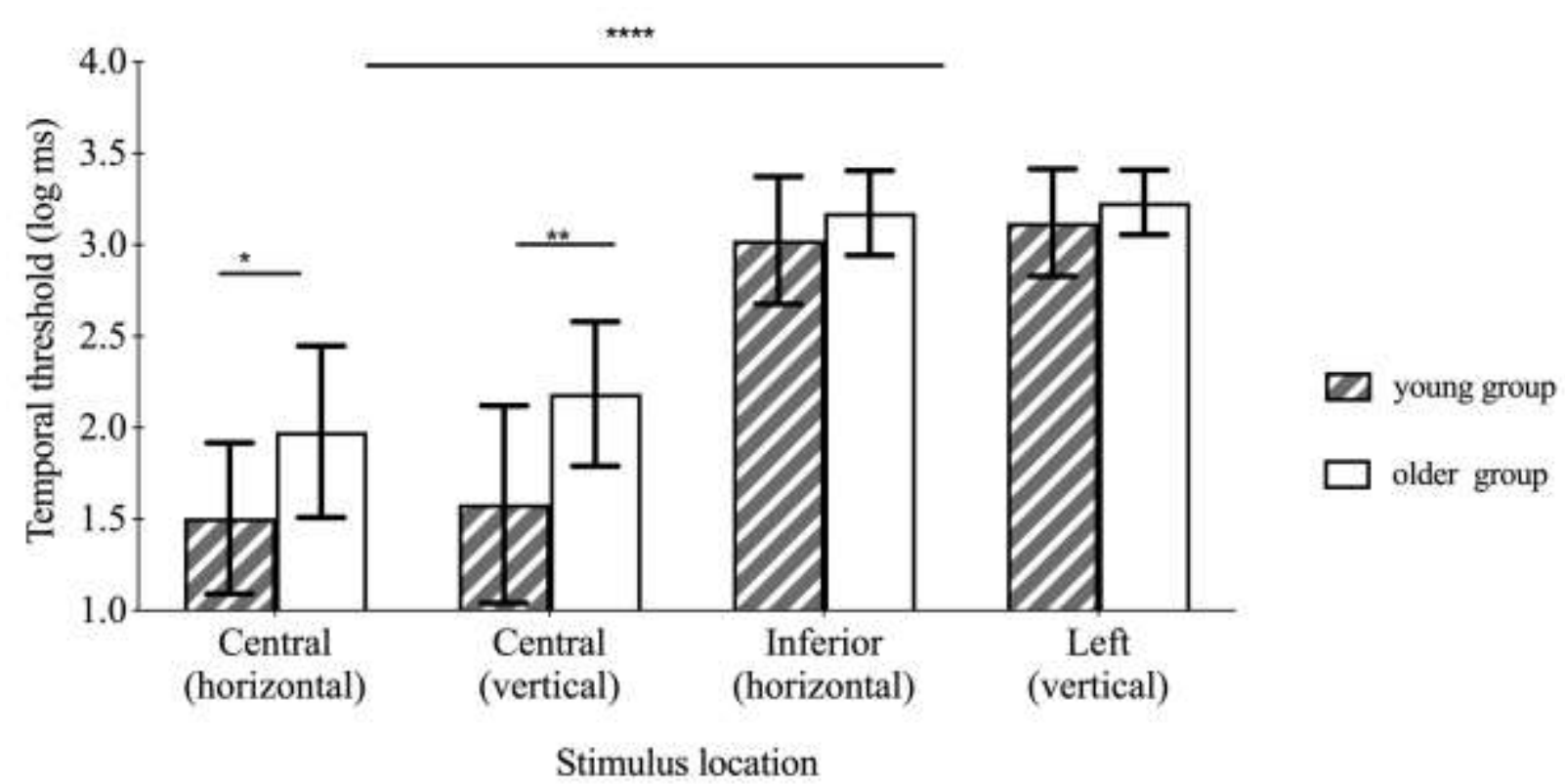

The temporal trigram thresholds (TTT) for recognizing stimuli presented at central (horizontally-and vertically-oriented), inferior (horizontally-oriented) and left (verticallyoriented) visual fields for young and older groups. Each bar represents the average TTT with \pm 1 standard deviation as the error bar. Black horizontal lines are the significant differences between eccentricities or the significant differences between groups: **: $p<0.01$; ***: $p<0.001$.

\section{$3.3 \quad$ Spatial visual span (SVS)}

Fig. 8 illustrates the SVS profiles at central and peripheral visual fields for one young and one normally-sighted older subject. Similar to English reading, the accuracy of recognizing Chinese characters peaked at position 0 (central position) and gradually reduced as the displayed position increased away from the centre. At central vision, the response function for recognizing horizontal trigrams had a higher peak than vertical trigrams (amplitude of $0.85 \pm 0.15$ vs. $0.79 \pm 0.14$ for horizontally- and vertically-presented trigrams respectively). The width of the SVS with function fitting at $80 \%$ correct was $2.97 \pm 1.22$ characters and $2.69 \pm 1.21$ (mean $\pm 1 \mathrm{SD}$ ) characters for recognizing horizontally-presented and vertically-presented trigrams respectively. At peripheral visual field, the peak amplitude reduced to approximately 0.4 to 0.5 , with narrower breadth. Hence, the width of SVS reduced to nearly 0 character at peripheral vision. In contrast to central vision, the profiles for the 
horizontally- and vertically-presented characters at peripheral vision varied individually, with some subjects showing a wider profile for recognizing trigrams presented at $10^{\circ}$ below fixation (Fig. 8 (a)), but vice versa for other subjects (Fig. 8 (b)).

Fig. 8 A sampled spatial visual span (SVS) profile for a young and an older subject
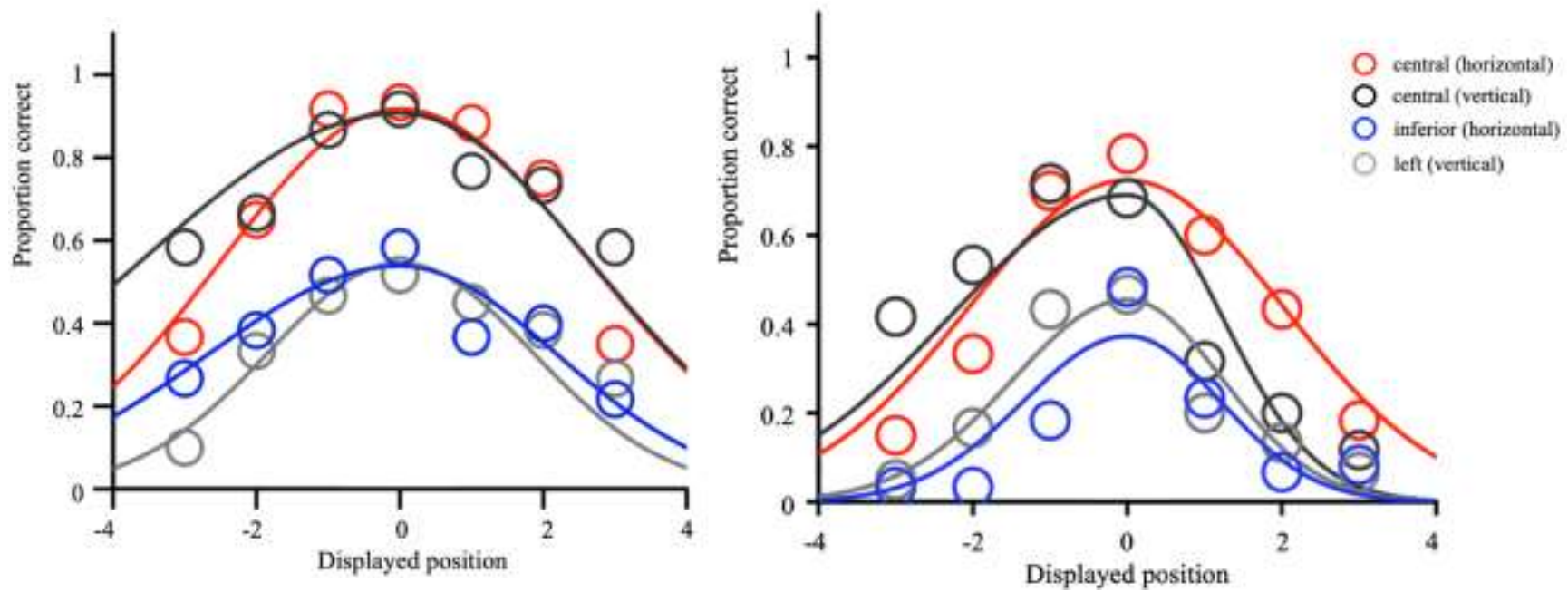

Individual SVS profile of a young subject (left panel) and an older subject (right panel) with normal vision. The red, black, blue and grey dots represent the accuracy for recognizing characters presented at each displayed position at central (horizontal-presented), central (vertically-presented), inferior (horizontally-presented) and left (vertically-presented) visual fields, respectively. Proportion of accuracy was combined across trials at each displayed position. Data for each SVS profile was fitted with split Gaussians.

Statistically, eccentricity $(F(1,24)=290.30, p<0.001)$, text orientation $(F(1,24)=9.28$, $\mathrm{p}=0.01)$, and age $(\mathrm{F}(1,24)=25.49, \mathrm{p}<0.001)$ all had significant main effects on the average percent correct of the central five character positions (Fig. 9). Significant interaction effects between eccentricity and text orientation $(F(1,24)=14.40, p<0.001)$, eccentricity and age $(\mathrm{F}(1,24)=10.52, \mathrm{p}=0.004)$, and age and text orientation $(\mathrm{F}(1,24)=6.08, \mathrm{p}=0.02)$ were observed. No interaction effect among eccentricity, age, and text orientation was observed $(F(1,24)=2.95, p=0.10)$. Higher recognition accuracy was observed when subjects read at central vision than peripheral vision ( $0.66 \pm 0.16$ vs. $0.31 \pm 0.09$ (mean $\pm 1 \mathrm{SD})$ ). Accuracy for recognizing horizontally-presented trigrams was slightly better than vertically-presented trigrams. However, the benefits of text orientation was only found at central vision $(0.68 \pm$ 0.16 vs. $0.63 \pm 0.15, p=0.03)$, but not at the peripheral locations $(0.31 \pm 0.07$ vs. $0.30 \pm 0.10$, 
$\mathrm{p}>0.99)$. Young subjects had higher accuracy than older subjects in both central $(0.76 \pm 0.12$ vs. $0.55 \pm 0.10, p<0.001)$ and peripheral visual locations $(0.35 \pm 0.10$ vs. $0.27 \pm 0.05$, $\mathrm{p}=0.006$ ). In addition, young subjects had a higher recognition accuracy when text was presented horizontally than vertically $(0.57 \pm 0.25$ vs. $0.54 \pm 0.23, \mathrm{p}=0.02)$, whereas such a difference was not shown in the older group $(0.41 \pm 0.19$ vs. $0.40 \pm 0.13, \mathrm{p}>0.99)$.

Fig. 9 Spatial visual span (SVS) at different visual conditions for young and older groups

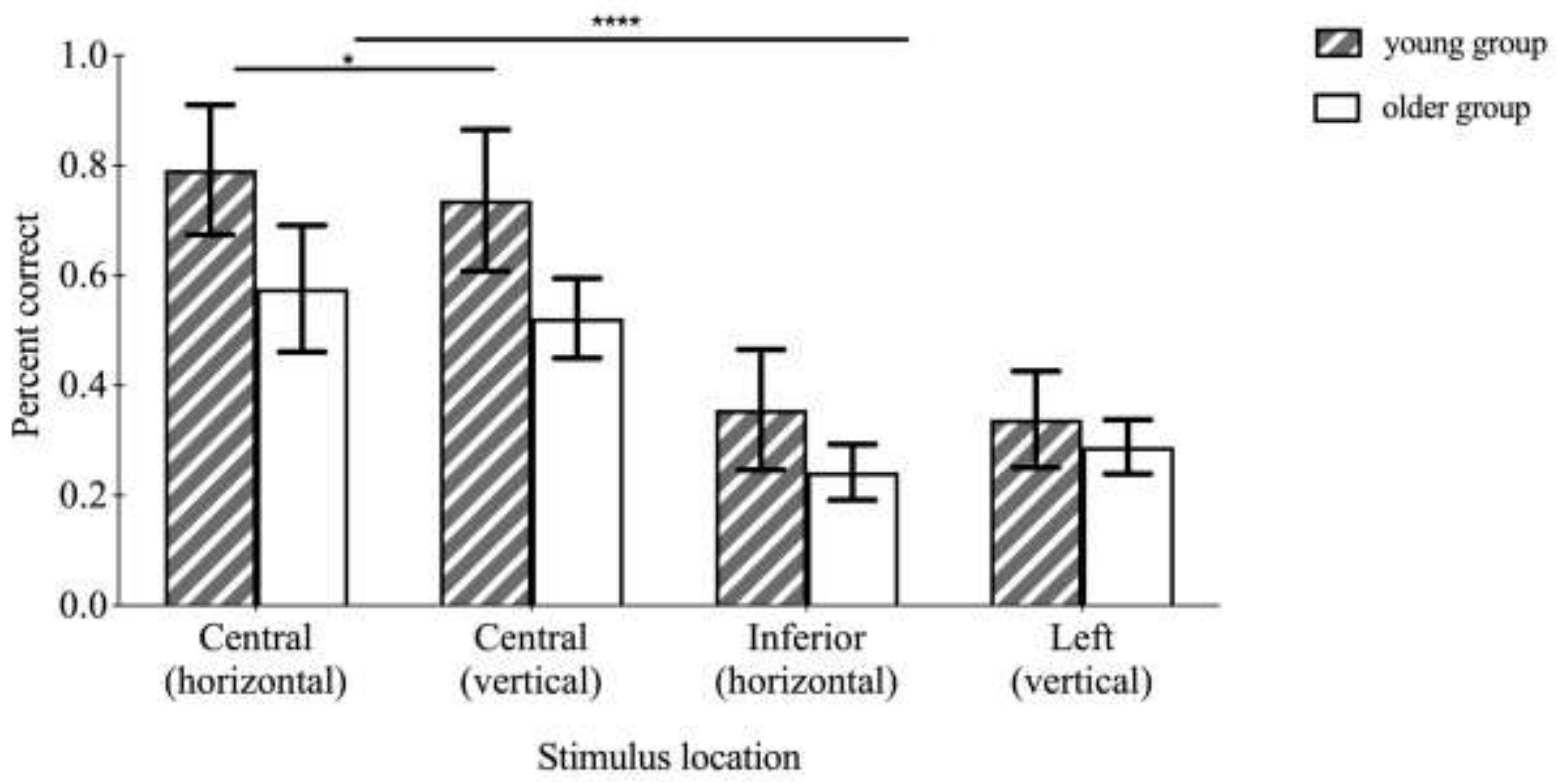

The average recognition accuracies in terms of the central 5-character positions with \pm 1 standard deviation for recognizing stimuli presented at central (horizontally-and verticallyoriented), inferior (horizontally-oriented) and left (vertically-oriented) visual fields for young and older groups. Black horizontal lines are the significant differences between eccentricities or the significant differences between text orientations.

To quantify the profile of visual span, the area under each fitted curve of SVS at central and peripheral locations was computed. Similar to the average recognition accuracy of the central five-character positions, eccentricity $(\mathrm{F}(1,24)=165.20, \mathrm{p}<0.001)$, text orientation $(F(1,24)=18.65, p<0.001)$, and age $(F(1,24)=19.72, p<0.001)$ showed significant main effects. Significant interaction effects between eccentricity and text orientation $(F(1,24)=7.73$, $\mathrm{p}=0.01)$ and between eccentricity and age $(\mathrm{F}(1,24)=12.34, \mathrm{p}=0.002)$ were observed. No interaction among eccentricity, age, and text orientation $(\mathrm{F}(1,24)=3.64, \mathrm{p}=0.07)$ or between age and text orientation $(\mathrm{F}(1,24)=2.34, \mathrm{p}=0.14)$ were observed. The AUC at central vision 
was significantly greater than that at peripheral vision $(0.40 \pm 0.14$ vs. $0.17 \pm 0.06$ (mean \pm 1 SD)). At central vision, characters presented horizontally had a greater AUC than those displayed vertically $(0.43 \pm 0.14$ vs. $0.38 \pm 0.14$, $\mathrm{p}<0.02)$, but no significant text orientation difference was found at the peripheral locations ( $0.17 \pm 0.04$ vs. $0.17 \pm 0.07, p>0.51)$. Young subjects had greater AUC than older subjects in both central (0.50 \pm 0.14 vs. $0.31 \pm 0.06$, $\mathrm{p}<0.001)$ and peripheral locations $(0.20 \pm 0.07$ vs. $0.14 \pm 0.03, \mathrm{p}=0.05)$.

\subsection{Relationships among temporal processing, visual span, and RSVP reading}

\section{performance at different stimulus locations}

The fitted regression model between visual performance and reading performance was analysed separately for central and peripheral visual fields (Table 2). At central vision, temporal processing in terms of $\log \mathrm{TTT}\left(\mathrm{R}^{2}=0.21\right.$ and $\mathrm{R}^{2}=0.17, \mathrm{p}<0.04$ for horizontal and vertical trigrams respectively) and SVS in terms of the average recognition accuracy of the central five character positions $\left(\mathrm{R}^{2}=0.30\right.$ and $\mathrm{R}^{2}=0.38, \mathrm{p}<0.01$ for horizontal and vertical trigrams respectively) could significantly predict the variance of RSVP MRS (Fig. 10). When correlating the reading speed with the entire spatial visual span profile, AUC could significantly predict MRS variance by $0.28(\mathrm{p}=0.005)$ and $0.44(\mathrm{p}<0.001)$ for horizontally and vertically presented characters respectively.

Table 2 Results of regression model between reading speed and visual span (Rsquared)

\begin{tabular}{|c|c|c|c|c|c|c|}
\hline \multirow[t]{2}{*}{$\begin{array}{l}\text { Reading } \\
\text { speed }\end{array}$} & \multicolumn{2}{|c|}{$\begin{array}{c}\text { Temporal processing } \\
\text { speed } \\
(\log \text { TTT })\end{array}$} & \multicolumn{2}{|c|}{$\begin{array}{c}\text { Spatial visual span } \\
\text { (Average recognition } \\
\text { accuracy) }\end{array}$} & \multicolumn{2}{|c|}{$\begin{array}{c}\text { Spatial visual span } \\
\text { (AUC) }\end{array}$} \\
\hline & Horizontal & Vertical & Horizontal & Vertical & Horizontal & Vertical \\
\hline $\begin{array}{l}\text { Central } \\
\text { visual field }\end{array}$ & $0.21 *$ & $0.17 *$ & $0.30 * *$ & $0.38 * *$ & $0.28 * *$ & $0.44 * * *$ \\
\hline $\begin{array}{l}\text { Left visual } \\
\text { field }\end{array}$ & I & 0.01 & I & $0.27 * *$ & I & $0.35 * *$ \\
\hline $\begin{array}{l}\text { Inferior } \\
\text { visual field }\end{array}$ & $0.18 *$ & I & $0.57 * * *$ & I & $0.64 * * *$ & I \\
\hline
\end{tabular}


At peripheral vision, both $\log \mathrm{TTT}\left(\mathrm{R}^{2}=0.18, \mathrm{p}=0.03\right)$, spatial recognition accuracy of the central five character positions $\left(\mathrm{R}^{2}=0.57, \mathrm{p}<0.001\right)$ and AUC $\left(\mathrm{R}^{2}=0.64, \mathrm{p}<0.001\right)$ could significantly predict MRS at $10^{\circ}$ below fixation. However, when the characters were presented at $10^{\circ}$ left of fixation, the only significant results were between MRS and SVS, including spatial recognition accuracy $\left(\mathrm{R}^{2}=0.27, \mathrm{p}=0.007\right)$ and $\mathrm{AUC}\left(\mathrm{R}^{2}=0.35, \mathrm{p}=0.002\right)$, whereas $\log$ TTT could not statistically predict the variance of RSVP MRS $\left(R^{2}=0.01, p=0.64\right)$ (as shown by the grey curve for left lateral location in Fig. 10a). 
Fig. 10 Fitted regression models between RSVP MRS and TTT and SVS

(a) Correlation between RSVP MRS and TTT

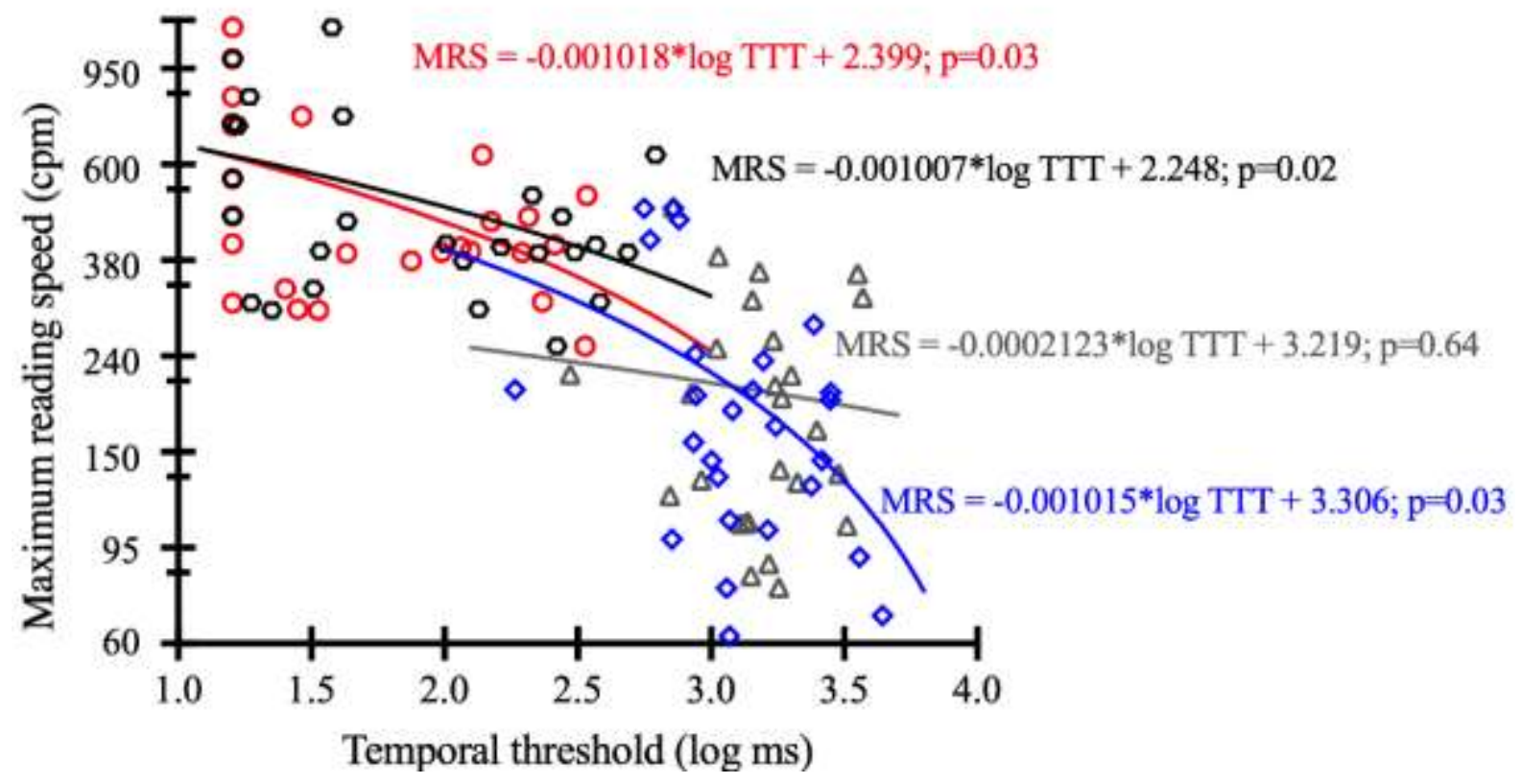

(b) Correlation between RSVP MRS and SVS

$-\operatorname{central}($ horizontal)

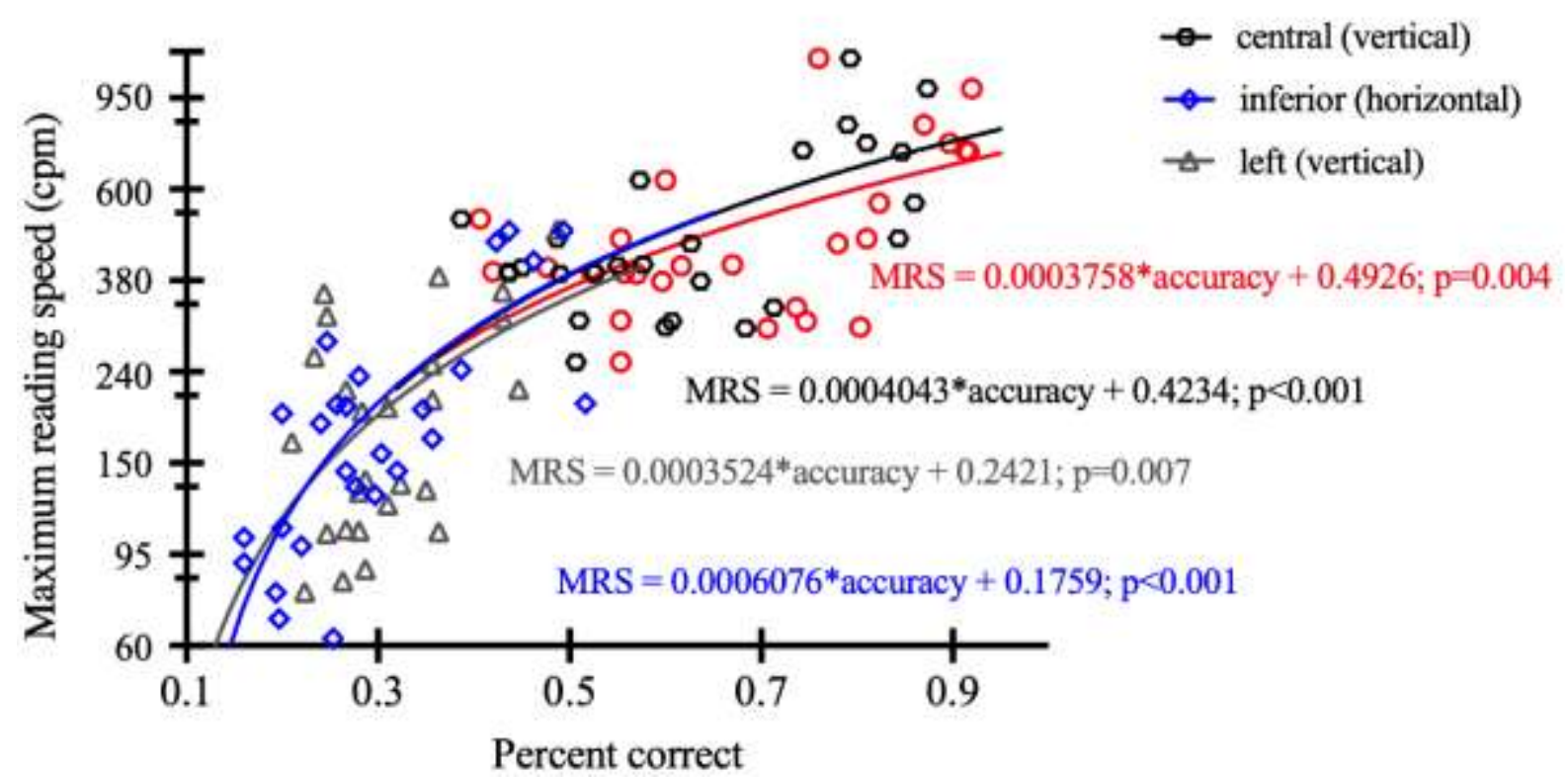

Fitted regression models between RSVP MRS and (a) TTT and (b) SVS in central and peripheral locations. Red circles represent individual log TTT or average recognition accuracy of the central five character positions for horizontally presented trigrams while black hexagons represent those for vertically presented characters at central vision. Blue diamonds and grey triangles represent individual log TTT or recognition accuracy at inferior and left peripheral presentations respectively. 


\section{Discussion}

To our knowledge, this is the first study to examine the visual factors associated with Chinese character reading performance at central and peripheral vision. Specifically, we aimed to examine 1) the correlation between visual span and reading of Chinese characters at normal central and peripheral vision; and 2) the effect of age on visual span and Chinese reading in healthy observers. First, it was found that RSVP reading speed at $10^{\circ}$ peripheral vision was approximately 2.5 times slower than central vision. In line with our hypothesis, longer temporal processing speed and smaller spatial visual span were correlated with the reduced reading speed at peripheral vision. Second, young subjects read roughly twice as fast as older subjects in all visual conditions, whereas mixed results were found for the effect of age on temporal processing and spatial visual span. Age-related decline in TTT was only found at central vision, but not at peripheral vision (Fig 7).

\subsection{Chinese character reading performance at central vs. peripheral vision}

Our results showed approximately a 2.5-fold decrease in maximum Chinese reading speeds from central to $10^{\circ}$ peripheral vision. Although Chinese characters are considered as more complex stimuli than alphabets because of the increased within-stimulus crowding (Wang et al., 2014), this eccentricity-decline in reading speed was less than those reported in studies among other languages using word-by-word RSVP presentation, implying less impact of eccentricities on Chinese characters reading. In English, young readers' RSVP reading speed showed a 2.8 to 3.7-fold decrease from central to $10^{\circ}$ below fixation (Chung et al., 1998; Yu, Cheung, Chung \& Legge, 2006). In contrast, a factor of 6.5 decline in reading speed at $10^{\circ}$ inferior visual field was found in Korean (Hangul), which shares a similar configuration to Chinese reading (He, Baek \& Legge, 2018). The slower Chinese RSVP reading speed at central vision ( 510 cpm vs. 787 and 713 wpm in English and Korean reading) might be one reason explaining the less significant decline in reading speed at peripheral vision. However, further study on a larger sample size is required to confirm the effect of eccentricity on Chinese reading speed. Furthermore, the current Chinese RSVP reading speed was examined using character-by-character presentation rather than word-byword presentation as in the English (Chung et al., 1998; Yu et al., 2006) and Korean studies (He et al., 2018), because Chinese characters can stand alone as a meaningful word. However, this reduces the potential between-character crowding effects when assessing Chinese RSVP reading performance. Further study presenting both within- and between- 
characters crowding RSVP measurement is required to compare Chinese reading performance with other languages at both central and peripheral visual fields.

\subsection{Spatial visual span and temporal processing speed at central vs. peripheral vision}

The results of the current study are consistent with previous studies observing that both spatial visual span (Chung et al., 2004; Yu et al., 2010) and temporal threshold (Cheong et al., 2007) significantly decreased from central to peripheral vision. Due to the different methodologies for measuring visual span, three approaches were used to compare our SVS findings with those of previous studies. First, previous studies demonstrated that the SVS for English letters was estimated to be 10 letters (Fine \& Rubin, 1999; Legge et al., 1997; Legge et al., 2001; Rayner \& Bertera, 1979) at central vision, and dropped to 1.7 letters at $15^{\circ}$ below fixation (Legge et al., 2001). Our study found that the estimated SVS for Chinese characters was 3.0 and 2.7 characters for horizontally- and vertically-presented trigrams at central vision. Given that all participants' SVS profiles at peripheral vision could not reach $80 \%$ accuracy or above, the estimated width then dropped to 0 character at $10^{\circ}$ peripheral vision. Second, a comparison of the average recognition accuracy for the central five character positions at central and peripheral vision (Fig 9) revealed that the accuracy dropped by 39\% and $32 \%$ for recognizing horizontally and vertically-presented trigrams, respectively, at inferior and left visual fields compared with central vision. Third, the area under the individual fitted Gaussian curves decreased by 0.25 and 0.21 from central to peripheral visual fields for horizontal and vertical characters respectively.

Opposing to our hypothesis, we did not find any significant difference between the horizontal and vertical trigrams presented at the inferior and left visual locations respectively. The lack of orientation difference could be attributed to different testing locations or to the different presentation orientations at the periphery. Zhang et al. revealed strong betweencharacter crowding for recognizing Chinese characters at peripheral vision (Zhang et al., 2009). However, such crowding effect was reduced as a result of "spatial complexity contrast" between the target and flanking characters. Since the current study adopted characters with various strokes, which were randomly selected to form a trigram, the different complexities between characters within the trigram might be one reason explaining the insignificant effect of text orientations. Future studies presenting both horizontal and vertical 
texts at the two peripheral locations could examine the effect of presented orientations on Chinese characters recognition performance at peripheral vision.

Although no previous studies have examined the Chinese SVS at peripheral vision, this study revealed a narrower SVS profile at central vision than those reported in previous studies. For example, Wang et al. reported that the estimated SVS for high, middle and low complexity characters was 4.5, 6.0 and 6.7 characters (Wang et al., 2014), while 5.5, 6.5 and 7.2 characters were reported in Xie and colleagues (Xie et al., 2019). Although the characters adopted in the current study might be regarded as "middle" complexity, the estimated SVS remained much smaller than previous studies. This could be explained by three discrepancies in the methodology. First, the three Chinese characters were randomly selected from a pool of 250 Chinese characters, decreasing the chance of guessing (1/250) compared to Chinese characters or English alphabets (1/26) adopted in previous studies. Second, instead of reporting the presented characters verbally because some characters might have similar pronunciation and/or configuration, participants were asked to identify each character in a panel of 20 characters presented on the monitor (Fig. 4 and 5). The greater between- and within-character crowding during additional "visual search" might further increase the difficulty of the recognition task, resulting in the smaller SVS in our study. Third, to minimize eye movements, a fixed exposure duration of $200 \mathrm{~ms}$ was used. However, such a short exposure duration might impose a stronger limitation in SVS because of the difficulty in processing peripheral visual stimuli, which is explained further below.

The TTT for recognizing English letters at normal peripheral vision was significantly longer than central vision (e.g. equal to or less than 13 vs. $97 \mathrm{~ms}$ for central and $10^{\circ}$ below fixation reported by Cheong et al. (2007) and $217 \mathrm{~ms}$ at $10^{\circ}$ inferior visual field by $\mathrm{Yu}$ et al. (2018)). Consistent with previous studies, the current study reported a greater decline of TTT for recognizing Chinese characters at peripheral vision than central vision. In addition to the more difficult recognition task adopted in our study (i.e. recognizing target characters among a panel of 20 characters), difficulty in recognizing Chinese characters at peripheral vision could be another reason explaining the longer processing time required. TTT increased by 96\% and 95\% for recognizing horizontally- and vertically-presented Chinese characters at inferior and left visual fields, but only $87 \%$ increase of TTT was required for peripheral English letter recognition (Cheong et al., 2007). Since Chinese characters comprise internal 
and external crowding, it remains unclear which factor(s) explain the slower processing speed of recognizing Chinese characters, especially at peripheral vision. A future study which examines the difference of visual span for recognizing an isolated Chinese character and 3Chinese characters (i.e. trigram) will provide further information about the impact of crowding on Chinese temporal processing speed at peripheral vision.

\subsection{Could spatial visual span and temporal processing speed explain Chinese reading}

\section{performance?}

In line with our hypothesis, both spatial visual span and temporal processing speed were correlated with Chinese reading speed at central and peripheral visual fields. SVS in terms of the average recognition accuracy and AUC explained 27 to $64 \%$ variances of Chinese reading speed (Table 2) at the corresponding eccentricity, while TTT explained 17 to $21 \%$ variances of reading speed (except $10^{\circ}$ left visual field). These findings were similar to those reported in previous studies. In English reading, SVS in terms of visual span size explained approximately 53 to $94 \%$ of reading speed across different studies (Chung et al., 1998; Legge et al., 2001; Yu, Cheung, Legge \& Chung, 2007), while TTT explained 58\% reading speed (Cheong et al., 2007) at normal peripheral vision ${ }^{\mathrm{b}}$.

As expected, a person with longer temporal processing speed read slower at central and inferior visual fields. However, the lack of association between TTT and Chinese reading speed at left peripheral viewing was surprising (Fig 10a). Chinese text can be oriented at both horizontal and vertical directions. However, most modern Chinese reading materials use the horizontal layout, so it was not surprising that participants spent more time reading Chinese text in horizontal than vertical dimensions $(>70 \%)$. However, this potential unfamiliarity of reading vertical text might not explain the dissociation of vertical character recognition and reading at left lateral vision because of no significant difference for reading/recognizing characters between different orientations at peripheral vision (Fig. 6, 7 and 9). An alternative explanation could be the "context gain" (i.e. the ratio of sentence to random words reading rate), which was found to be greater at left lateral peripheral vision, compared to central and inferior visual fields (Fine, Hazel, Petre \& Rubin, 1999). Hence cognitive capacity, rather

\footnotetext{
${ }^{\mathrm{b}}$ Reading speed and visual span at normal and peripheral vision was examined in a small sample size of 5 subjects in Cheong et al. (2007).
} 
than visual factors, may play a more important role in limiting reading speed at left peripheral visual field.

\subsection{Effect of age on Chinese reading performance}

Consistent with previous studies in English, it was found that young subjects read faster at both central and peripheral vision (Yu et al., 2006). Compared with Yu et al.'s result, there was a stronger age-related decline (2 vs. 1.5 times slower) in Chinese character-bycharacter reading for peripheral vision than central vision. This might be due to a stronger crowding effect at peripheral vision, in particular for Chinese reading, explaining the slower reading speed in older adults (Liu, Patel \& Kwon, 2017). Given the significant association of temporal processing speed, spatial visual span, and reading speed, it should be expected that there would be a similar age-related decline in TTT and SVS. Unexpectedly, the age-related decline was mainly noted at central vision, rather than peripheral vision. Young subjects showed shorter TTT and higher SVS than older subjects at central vision (Warrington, Xie, Wang \& Paterson, 2019; Xie et al., 2019). As half of the young subjects' TTT was equal to or exceeded the refresh limitation of the displayed monitor for recognizing horizontally orientated stimuli presented at central vision, while only two older subjects achieved $80 \%$ or above correct at the shortest exposure time of $16.7 \mathrm{~ms}$, this reflects an even greater TTT difference between ages at the central visual field. The sensory decline in older adults limits the amount of information processed in each glance, requiring longer time to process the information (Warrington et al., 2019). Despite a stronger crowding effect in peripheral vision, the decline of SVS and TTT at peripheral vision was weaker in older adults. Unlike RSVP reading with only one character presented, a three-character trigram was used to measure the SVS and TTT. It is possible that the "between-character" crowding effect at peripheral vision might vary across age groups, implying that peripheral vision in older adults might be more susceptible to within-character crowding than between-character crowding. However, this speculation requires further study.

\subsection{Limitations of the study}

The current study has several limitations. First, young subjects on average had a higher educational level than the older subjects, although the stimuli used in the study were within the subjects' literacy skills. Having older subjects from a more common educational background could better reflect this age group's reading performance in the community, allowing a direct comparison with reading performance in visually impaired patients of 
similar age groups. Future studies could recruit subjects with more balanced educational backgrounds and a wider range of ages to investigate the effect of age on central and peripheral Chinese reading ability. Second, due to the limitations of the displayed monitor's refresh rate, the shortest exposure duration for TTT characters presentation was $16.7 \mathrm{~ms}$. The potential impact of this limitation has been explained above in section 4.4.

\section{Conclusions}

This study contributed novel insights into Chinese reading performance at healthy peripheral vision and demonstrated the significant association among temporal processing speed, spatial visual span, and Chinese reading performance at healthy central and inferior visual fields. Understanding Chinese peripheral reading performance in participants with normal vision may help generate better rehabilitation solutions for the low vision community, who depend primarily on peripheral vision.

\section{Acknowledgments}

We thank Dr. Maureen Boost for the critical reading of the manuscript. This research was supported in part by grants from The Hong Kong Polytechnic University (internal grant UAG2 to AC and postgraduate research studentship to AQL) and Velux Stiftung Foundation (Grant 1188 to $\mathrm{AC}$ and BT). Preliminary findings were presented as a poster at the Association for Research in Vision and Ophthalmology 2021.

Declarations of interest: none.

\section{References}

Bethlehem, R.A., Dumoulin, S.O., Dalmaijer, E.S., Smit, M., Berendschot, T.T., Nijboer, T.C., \& Van der Stigchel, S. (2014). Decreased fixation stability of the preferred retinal location in juvenile macular degeneration. PLoS One, 9 (6), e100171.

Bullimore, M.A., \& Bailey, I.L. (1995). Reading and eye movements in age-related maculopathy. Optom Vis Sci, 72 (2), 125-138.

Cheong, A.M., Legge, G.E., Lawrence, M.G., Cheung, S.H., \& Ruff, M.A. (2007). Relationship between slow visual processing and reading speed in people with macular degeneration. Vision Res, 47 (23), 2943-2955. 
Cheong, A.M., Legge, G.E., Lawrence, M.G., Cheung, S.H., \& Ruff, M.A. (2008).

Relationship between visual span and reading performance in age-related macular degeneration. Vision Res, 48 (4), 577-588.

Chung, S.T. (2002). The effect of letter spacing on reading speed in central and peripheral vision. Invest Ophthalmol Vis Sci, 43 (4), 1270-1276.

Chung, S.T. (2010). Enhancing visual performance for people with central vision loss. Optom Vis Sci, 87 (4), 276-284.

Chung, S.T. (2011). Improving reading speed for people with central vision loss through perceptual learning. Invest Ophthalmol Vis Sci, 52 (2), 1164-1170.

Chung, S.T., Legge, G.E., \& Cheung, S.H. (2004). Letter-recognition and reading speed in peripheral vision benefit from perceptual learning. Vision Res, 44 (7), 695-709.

Chung, S.T., Mansfield, J.S., \& Legge, G.E. (1998). Psychophysics of reading. XVIII. The effect of print size on reading speed in normal peripheral vision. Vision Res, 38 (19), 29492962.

Chung, S.T. (2021). Training to improve temporal processing of letters benefits reading speed for people with central vision loss. $J$ Vis, 21 (1), 14.

Coates, D.R., Chin, J.M., \& Chung, S.T. (2013). Factors affecting crowded acuity: eccentricity and contrast. Optom Vis Sci, 90 (7), 628-638.

Feng, C., Jiang, Y., \& He, S. (2007). Horizontal and vertical asymmetry in visual spatial crowding effects. $J$ Vis, 7 (2), 13.11-10.

Fine, E.M., Hazel, C.A., Petre, K.L., \& Rubin, G.S. (1999). Are the benefits of sentence context different in central and peripheral vision? Optom Vis Sci, 76 (11), 764-769.

Fine, E.M., \& Rubin, G.S. (1999). Reading with central field loss: number of letters masked is more important than the size of the mask in degrees. Vision Res, 39 (4), 747-756.

Fletcher, D.C., \& Schuchard, R.A. (1997). Preferred retinal loci relationship to macular scotomas in a low-vision population. Ophthalmology, 104 (4), 632-638.

He, Y., Baek, S., \& Legge, G.E. (2018). Korean reading speed: Effects of print size and retinal eccentricity. Vision Res, 150, 8-14.

Jacobs, R.J. (1979). Visual resolution and contour interaction in the fovea and periphery. Vision Res, 19 (11), 1187-1195.

Jin, G., Zou, M., Chen, A., Zhang, Y., Young, C.A., Wang, S.B., \& Zheng, D. (2019). Prevalence of age-related macular degeneration in Chinese populations worldwide: A systematic review and meta-analysis. Clin Exp Ophthalmol, 47 (8), 1019-1027. 
Kwon, M., Legge, G.E., \& Dubbels, B.R. (2007). Developmental changes in the visual span for reading. Vision Res, 47 (22), 2889-2900.

Lee, H.W., Legge, G.E., \& Ortiz, A. (2003). Is word recognition different in central and peripheral vision? Vision Res, 43 (26), 2837-2846.

Legge, G.E., Ahn, S.J., Klitz, T.S., \& Luebker, A. (1997). Psychophysics of reading--XVI. The visual span in normal and low vision. Vision Res, 37 (14), 1999-2010.

Legge, G.E., Cheung, S.H., Yu, D., Chung, S.T., Lee, H.W., \& Owens, D.P. (2007). The case for the visual span as a sensory bottleneck in reading. $J$ Vis, 7 (2), 9.1-15.

Legge, G.E., Hooven, T.A., Klitz, T.S., Stephen Mansfield, J.S., \& Tjan, B.S. (2002). Mr.

Chips 2002: new insights from an ideal-observer model of reading. Vision Res, 42 (18), 22192234.

Legge, G.E., Mansfield, J.S., \& Chung, S.T. (2001). Psychophysics of reading. XX. Linking letter recognition to reading speed in central and peripheral vision. Vision Res, 41 (6), 725 743.

Liu, R., Patel, B.N., \& Kwon, M. (2017). Age-related changes in crowding and reading speed. Sci Rep, 7 (1), 8271.

Octave, G.N.U. (2018). version 4.2. 2 manual: a high-level interactive language for numerical computations, John W. Eaton, David Bateman, Soren Hauberg, Rik Wehbring,

Peirce, J., \& MacAskill, M. (2018). Building experiments in PsychoPy. (Sage.

Rayner, K., \& Bertera, J.H. (1979). Reading without a fovea. Science, 206 (4417), 468-469.

Wang, H., He, X., \& Legge, G.E. (2014). Effect of pattern complexity on the visual span for Chinese and alphabet characters. J Vis, 14 (8), 6.

Wang, Y., Ng, A.L.K., Lai, J., \& Wong, I. (2019). prevalence of age-related macular degeneration in the adult population in Hong Kong: The Hong Kong Eye Survey. Invest Ophthalmol Vis Sci, 60 (9), 3963-3963.

Warrington, K.L., Xie, F., Wang, J., \& Paterson, K.B. (2019). Aging effects on the visual span for alphabetic stimuli. Exp Aging Res, 45 (5), 387-399.

Whittaker, S.G., Cummings, R.W., \& Swieson, L.R. (1991). Saccade control without a fovea. Vision Res, 31 (12), 2209-2218.

Wichmann, F.A., \& Hill, N.J. (2001a). The psychometric function: I. Fitting, sampling, and goodness of fit. Percept Psychophys, 63 (8), 1293-1313.

Wichmann, F.A., \& Hill, N.J. (2001b). The psychometric function: II. Bootstrap-based confidence intervals and sampling. Percept Psychophys, 63 (8), 1314-1329. 
Xie, F., Li, L., Zhao, S., Wang, J., Paterson, K.B., White, S.J., \& Warrington, K.L. (2019). Aging and pattern complexity effects on the visual span: evidence from Chinese Character recognition. Vision (Basel), 3 (1)

Yeung, PS., Ho, C.S.H., Chik, P.P.M., Lo, L.Y.., Luan, H., Chan, D.W.O., \& Chung, K.K.H. (2011). Reading and spelling Chinese among beginning readers: What skills make a difference? Sci. Stud. Read, 15 (4), 285-313.

Yu, D., Cheung, S.H., Chung, S.T., \& Legge, G.E. (2006). Age effects on reading speed and visual span in peripheral vision. $J$ Vis, 6 (6), 1001-1001.

Yu, D., Cheung, S.H., Legge, G.E., \& Chung, S.T. (2007). Effect of letter spacing on visual span and reading speed. $J$ Vis, 7 (2), 2.1-10.

Yu, D., Cheung, S.H., Legge, G.E., \& Chung, S.T. (2010). Reading speed in the peripheral visual field of older adults: Does it benefit from perceptual learning? Vision Res, 50 (9), 860869.

Yu, D., Legge, G.E., Wagoner, G., \& Chung, S.T. (2018). Training peripheral vision to read: Boosting the speed of letter processing. Vision Res, 152, 51-60.

Zhang, J.Y., Zhang, T., Xue, F., Liu, L., \& Yu, C. (2009). Legibility of Chinese characters in peripheral vision and the top-down influences on crowding. Vision Res, 49 (1), 44-53.

Zhu, Z., Hu, Y., Liao, C., Huang, R., Keel, S., Liu, Y., \& He, M. (2019a). Perceptual learning of visual span improves Chinese reading speed. Invest Ophthalmol Vis Sci, 60 (6), 23572368 .

Zhu, Z., Hu, Y., Liao, C., Keel, S., Huang, R., Liu, Y., \& He, M. (2019b). Visual span and cognitive factors affect Chinese reading speed. $J$ Vis, 19 (14), 17. 
Appendix I: Results of a mini-validation study of 605 sentences used in the study

\begin{tabular}{|l|l|l|l|l|}
\hline Subject ID & Age (years) & Educational level & $\begin{array}{l}\text { Reading speed } \\
(\mathrm{wpm})\end{array}$ & Total errors \\
\hline Young 001 & 25.53 & University or above & $246.03 \pm 43.22$ & 35 \\
\hline Young 002 & 21.88 & University or above & $200.20 \pm 40.85$ & 53 \\
\hline Young 003 & 33.24 & University or above & $213.70 \pm 33.24$ & 41 \\
\hline Young 004 & 21.45 & University or above & $278.72 \pm 47.65$ & 14 \\
\hline Young 005 & 30.72 & University or above & $266.05 \pm 36.82$ & 14 \\
\hline Young 006 & 25.04 & University or above & $216.90 \pm 24.84$ & 8 \\
\hline Young 007 & 30.00 & University or above & $203.13 \pm 18.76$ & 19 \\
\hline Elderly 001 & 64.07 & University or above & $183.22 \pm 20.74$ & 20 \\
\hline Elderly 002 & 67.62 & University or above & $145.30 \pm 17.97$ & 9 \\
\hline Elderly 003 & 72.49 & University or above & $190.29 \pm 30.36$ & 20 \\
\hline Elderly 004 & 61.28 & Secondary school & $187.72 \pm 29.17$ & 16 \\
\hline Elderly 005 & 63.79 & Secondary school & $175.58 \pm 25.52$ & 27 \\
\hline Elderly 006 & 73.67 & Primary school & $181.05 \pm 25.25$ & 17 \\
\hline Elderly 007 & 67.93 & Primary school & $210.38 \pm 25.19$ & 14 \\
\hline
\end{tabular}

A group of 7 normally sighted young (mean age of $26.84 \pm 4.56$ years ( 1 SD) and 7 elderly subjects (mean age of $67.26 \pm 4.60$ years) were recruited to complete a mini-validation study by reading the tested sentences which were presented in a single line in random order.

Participants were asked to read the sentences aloud as quickly as possible. The font size was $1.2 \log M A R$. Young subjects had a quicker reading speed than the elderly subjects (mean of $232.10 \pm 31.48$ vs. $181.93 \pm 19.57 \mathrm{cpm}($ mean $\pm 1 S D), t(12)=3.58, p=0.004)$. However, similar numbers of errors were made across the groups (26.29 \pm 16.81 vs. $17.57 \pm 5.62$, $t(12)=1.30, p=0.22)$. 